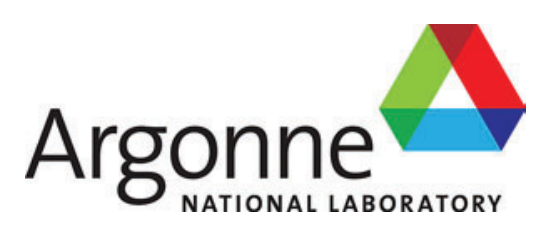

ANL-21/42

\title{
2020 LDRD Annual Report
}

Laboratory Directed Research and Development Program Activities

Laboratory Directed Research and Development 
This material is based upon work supported by Laboratory Directed Research and Development (LDRD)

funding from Argonne National Laboratory, provided by the Director, Office of Science, of the U.S.

Department of Energy under Contract No. DE-AC02-06CH11357

\author{
About Argonne National Laboratory \\ Argonne is a U.S. Department of Energy laboratory managed by UChicago Argonne, LLC \\ under contract DE-AC02-06CH11357. The Laboratory's main facility is outside Chicago, \\ at 9700 South Cass Avenue, Argonne, Illinois 60439. For information about Argonne \\ and its pioneering science and technology programs, see www.anl.gov.
}

\title{
DOCUMENT AVAILABILITY
}

Online Access: U.S. Department of Energy (DOE) reports produced after 1991 and a growing number of pre-1991 documents are available free at OSTI.GOV (http://www.osti.gov/), a service of the U.S. Dept. of Energy's Office of Scientific and Technical Information

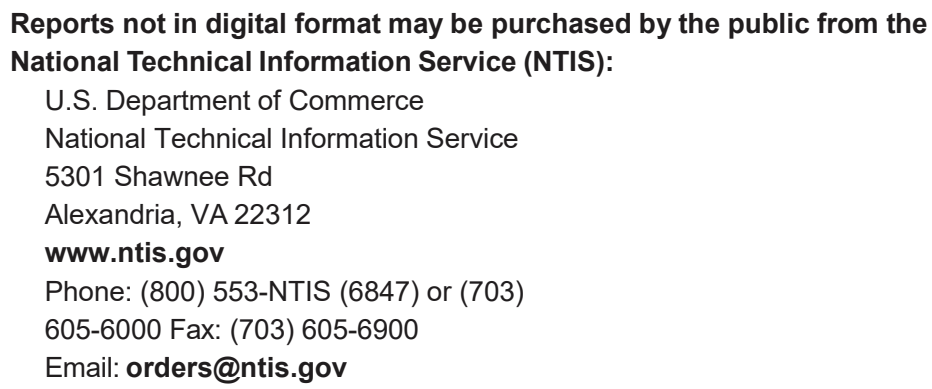

Reports not in digital format are available to DOE and DOE contractors from the Office of Scientific and Technical Information (OSTI):

U.S. Department of Energy

Office of Scientific and Technical Information

P.O. Box 62

Oak Ridge, TN 37831-0062

www.osti.gov

Phone: (865) 576-8401

Fax: (865) 576-5728

Email: reports@osti.gov

\section{Disclaimer}

This report was prepared as an account of work sponsored by an agency of the United States Government. Neither the United States Government nor any agency thereof, nor UChicago Argonne, LLC, nor any of their employees or officers, makes any warranty, express or implied, or assumes any legal liability or responsibility for the accuracy, completeness, or usefulness of any information, apparatus, product, or process disclosed, or represents that its use would not infringe privately owned rights. Reference herein to any specific commercial product, process, or service by trade name, trademark, manufacturer, or otherwise, does not necessarily constitute or imply its endorsement, recommendation, or favoring by the United States Government or any agency thereof. The views and opinions of document authors expressed herein do not necessarily state or reflect those of the United States Government or any agency thereof, Argonne National Laboratory, or UChicago Argonne, LLC. 
ANL-21/42

\section{LDRD Annual Report}

\section{Laboratory Directed Research and Development Program Activities}

prepared by

Vivian Sullivan, LDRD Program Manager, Office of Strategy, Performance, and Risk;

Office of the Director

Laboratory Directed Research and Development, Argonne National Laboratory

September 1, 2021 


\title{
LDRD Program Overview
}

Argonne National Laboratory's Laboratory Directed Research and Development (LDRD) program encourages the development of novel technical concepts, enhances the Laboratory's research and development (R\&D) capabilities, and enables pursuit of strategic laboratory goals.

Argonne's LDRD projects are proposal based and peer reviewed, supporting ideas that require advanced exploration so they can be sufficiently developed to pursue support through normal programmatic channels. Among the aims of the projects supported by the LDRD program are the establishment of engineering proofs of principle, assessment of design feasibility for prospective facilities, development of instrumentation or computational methods or systems, and discoveries in fundamental science and exploratory development.

All LDRD projects have demonstrable ties to one or more of the science, energy, environment, and national security missions of the U.S. Department of Energy (DOE) and its National Nuclear Security Administration (NNSA), and many are also relevant to the missions of other federal agencies that sponsor work at Argonne. A natural consequence of the more "applied" type projects is their concurrent relevance to industry.

The LDRD program is managed in overarching portfolios, each containing multiple projects each fiscal year. The LDRD Prime portfolio is further divided into strategic focus areas aligned with Argonne's strategic plan.

\section{FY 2020 LDRD Program Components}

\section{LDRD Prime}

The largest component of Argonne's program is LDRD Prime, which emphasizes R\&D explicitly aligned with Laboratory major initiatives in support of Argonne's strategic plan. The choice of Focus Areas under the LDRD Prime component reflects the major initiatives; the state of development of relevant technical fields; the potential value of advancing those fields to DOE/NNSA and the nation; and the compatibility of the fields with existing facilities, capabilities, and staff expertise at Argonne.

Focus Areas with projects that ended in FY20 are:

\author{
Advanced Computing \\ Al + Science \\ Biological and Environmental Science \\ Energy Storage \\ Hard X-ray Sciences \\ Manufacturing Science and Engineering \\ Materials and Chemistry \\ Physical Sciences and Engineering \\ Quantum Information Sciences \\ Securing Energy and Critical Resources \\ The Universe as Our Laboratory (ULab)
}




\section{Director's Collaborations}

The Director's Collaborations LDRD projects support research that is paired with coordinating research efforts at a partner institution. These are generally small projects selected through a collaborative process.

\section{Named Fellows}

Argonne's LDRD Named Fellows program aims to support the scientific or engineering research of exceptional early career scientists and engineers. Working with an Argonne sponsor (a senior member of research staff), LDRD Named Fellows carry out work that is either at the forefront of new research areas or is synergistic with current research efforts.

\section{Innovate}

The Innovate component of the LDRD program invests in a full spectrum of investigator-initiated proposals across the Laboratory in DOE-mission-related science and engineering areas. This provides an avenue for R\&D staff to propose highly innovative projects in research areas outside the purview of the Prime Focus Areas. Within the Innovate LDRD portfolio is the Seed program, open to postdoctoral appointees and early career researchers to apply for a small project to perform independent research and explore their own ideas.

\section{Swift}

The LDRD Swift component provides an avenue for R\&D staff to conduct short-term research with a targeted funding opportunity in mind, as well as a means for researchers to explore ideas before developing a full proposal. Projects funded through this component area have a maximum one-year duration. As with the Innovate component, the Swift component invests in a full spectrum of proposals across all mission-related science and engineering areas.

\section{FY 2020 LDRD Summary Report}

This summary report provides an overview of all LDRD projects at Argonne that concluded in Fiscal Year 2020. Many projects are funded for multiple years, the initial fiscal year for each project is indicated by the first four digits of the LDRD project number. 


\begin{tabular}{|c|c|c|c|}
\hline $\begin{array}{l}\text { LDRD Project } \\
\text { Number }\end{array}$ & Title & Lead PI(s) & Page \\
\hline \multicolumn{4}{|c|}{ ADVANCED COMPUTING } \\
\hline 2018-058 & $\begin{array}{l}\text { Modeling and Quantifying Tradeoffs among Execution } \\
\text { Time, Power, and Resilience of Parallel Application to } \\
\text { Efficiently Exploit System Heterogeneity }\end{array}$ & Valerie Taylor & 1 \\
\hline 2019-0030 & $\begin{array}{l}\text { Probabilistic Forecasting of Extreme Weather Using } \\
\text { Deep Learning }\end{array}$ & Carlo J. Graziani & 1 \\
\hline 2019-0202 & $\begin{array}{l}\text { Development and Application of Advanced Computing } \\
\text { Technologies and Models for Hypersonic Combustion } \\
\text { Applications }\end{array}$ & Sibendu Som & 2 \\
\hline 2019-0300 & $\begin{array}{l}\text { Enabling Automatic Learning of Atmospheric Particles } \\
\text { through APS-U }\end{array}$ & Zichao Di & 3 \\
\hline 2019-0313 & Neuromorphic Learning for Neuroimaging & Fangfang Xia & 3 \\
\hline $2020-0473$ & Advanced Computing Expedition 2020 & Michael Papka & 4 \\
\hline \multicolumn{4}{|c|}{$\mathrm{Al}+\mathrm{SCIENCE}$} \\
\hline $2020-0168$ & Al Robotics for Solution-Processable Materials & Pierre T. Darancet & 5 \\
\hline $2020-0169$ & $\begin{array}{l}\text { Al-driven Design of Controlled Phase Separation } \\
\text { Mediated by Intrinsically Disordered Peptide } \\
\text { Polymers }\end{array}$ & Arvind Ramanathan & 5 \\
\hline 2020-0170 & $\begin{array}{l}\text { Building an Autonomous Materials Discovery (AMD) } \\
\text { System for Metamaterials and the Associated Data } \\
\text { Infrastructure }\end{array}$ & Benjamin J. Blaiszik & 6 \\
\hline \multicolumn{4}{|c|}{ BIOLOGICAL AND ENVIRONMENTAL SCIENCE } \\
\hline 2018-055 & $\begin{array}{l}\text { Variability and Trends in Land Surface Properties: } \\
\text { Effects on Boundary Layer Physics and Extremes } \\
\text { Onset }\end{array}$ & Yan Feng & 6 \\
\hline 2019-0340 & $\begin{array}{l}\text { Design and Test Synthetic Pathways to Optimize their } \\
\text { Effectiveness and Produce New Chemical Diversity }\end{array}$ & Michael Fonstein & 7 \\
\hline \multicolumn{4}{|c|}{ ENERGY STORAGE } \\
\hline 2018-100 & $\begin{array}{l}\text { High-Density Energy Storage in Solvent-Free Redox } \\
\text { Flow Batteries for Flexible Electric Grid }\end{array}$ & Lu Zhang & 8 \\
\hline 2018-104 & $\begin{array}{l}\text { Complex Fluids from Chemical Separations: A New } \\
\text { Entry to Battery Electrolytes }\end{array}$ & Lynda Soderholm & 9 \\
\hline 2018-105 & $\begin{array}{l}\text { Electrode Contamination Investigation to Advance } \\
\text { Direct Recycling }\end{array}$ & Jeffrey Spangenberger & 9 \\
\hline \multicolumn{4}{|c|}{ HARD X-RAY SCIENCES } \\
\hline $2018-002$ & $\begin{array}{l}\text { Development of a Hard X-ray Spectrometer Based on } \\
\text { Transition Edge Sensors for Advanced Spectroscopy }\end{array}$ & Orlando Quaranta & 10 \\
\hline 2018-016 & $\begin{array}{l}\text { Development of Zoom Optics Using Deformable } \\
\text { Mirrors with in situ Wavefront Sensing and a Closed- } \\
\text { loop Control System }\end{array}$ & Lahsen Assoufid & 11 \\
\hline
\end{tabular}


2018-019

2018-027

2018-028

2018-030

2018-054

2019-0028

\section{MANUFACTURING SCIENCE AND ENGINEERING}

2019-0026

Establishment of an Electrospinning-based Nanofiber Manufacturing Platform using in situ SAXS/WAXS

Measurements

2019-0254

Near Real-Time Optimization of Manufacturing

Processes via Machine Learning

2019-0279

2019-0330
Develop Spectroscopy and Imaging Capabilities for Microfluidic Channels

Integrated Sensor Array and Modeling for Rapid

Process Development in Chemical Vapor Deposition
Mathew Cherukara

11

Zahirul Islam

12

Jin Wang

Doga Gursoy

14

Alireza Nassiri

15

Yury Ivanyushenkov

Yuepeng Zhang

Marius Stan

16

Xiao-Min Lin

Jeffrey W. Elam

18

\section{MATERIALS AND CHEMISTRY}

2019-0205 Ion Transport in Heterogeneous Materials

2019-0433 Coupling Data Science and in situ Multi-Modal Probes for Materials Synthesis

G. Brian Stephenson 18 Richard E. Wilson

PHYSICAL SCIENCES AND ENGINEERING

2019-0386 Mineralogy Inspires Physics

2020-0164 Accelerators Against Cancer

Michael R. Norman

20

Jerry A. Nolen

20

QUANTUM INFORMATION SCIENCES

2019-0358 A Scalable Toolkit for Noisy-Qubit Simulation

2019-0360

Quantum State Engineering in Strong-Coupling

Circuits using Floquet Theory

2019-0377 Long-Lived Erbium Qubits in Silicon-Based

Semiconductor Materials

2019-0381 A Novel Readout for Large Arrays of Superconducting Nanowire Single Photon Detectors
Hal J. Finkel

21

Ivar Martin

21

Tijana Rajh

22

Clarence L. Chang 


\section{SECURING ENERGY AND CRITICAL RESOURCES \\ 2018-089 Optimizing Urban Transportation Systems Energy Using Large-Scale Simulation and Machine Learning \\ 2018-097 Mission-Driven Unmanned Aerial System (UAS) Design Center}

\section{THE UNIVERSE AS OUR LABORATORY ULAB)}

2018-053 A High Pressure Xenon Gas Time Projection Chamber for Next Generation Neutrinoless Double Beta Decay Search Experiments

2019-0034 Cooling the Cooler: Using Optical Stochastic Cooling to Prepare Cold Electrons for Cooling Hadrons in the Electron-Ion Collider

2019-0050 Horn-Coupled Detectors for Future CMB Experiments

Alexander A. Zholents

Thomas W. Cecil

\section{DIRECTOR'S COLLABORATIONS}

2018-110 Negative Capacitance in Ferroelectric Nanostructures

2020-0172 Computationally Evolved 2D Peptide Assemblies

Saidur Rahman Bakaul $\quad 27$

Harry C. Fry 28

\section{NAMED FELLOWS}

2017-093 Beehive: A Dynamic Execution Environment for
Performance, Power, and Resilience on Extreme-Scale

Min Si

Computing Systems

2017-157

Transport and Photophysical Processes in Hybrid

Peijun Guo

Perovskites for Energy Applications

2017-158 Real-time Control of Urban Drainage Systems

Sara Rimer $\quad 30$

2018-051 High-Efficiency, Low-cost ORR \& OER Catalysts Derived from Metal-Organic Frameworks

2018-052 Exploring Lossy Quantum Computation

Lina Chong

Matthew Otten based Method for Biocatalyst Engineering

Anand Bhattacharya

2020-0174 Semimetals

Tejas Guruswamy ray Transition Edge Sensor Arrays

Zhi Qiao 
2020-0182 In-situ Physics-of-Failure Analysis for Wide Bandgap

Power Device for Aerospace and Defense Application

2020-0194 Better Adversarial Machine Learning Through Robust Optimization

2020-0196

Artificial Intelligence-based Local Downscaling of

Global Coastal Flood Prediction

2020-0197 Statistics and Machine Learning to Enhance Reduced

Order Models

2020-0203 Modeling Dynamics of Complex Chemical Kinetics

using Neural Ordinary Differential Equations

2020-0204

2020-0208

2020-0214

2020-0218

2020-0225

$2020-0226$

2020-0229

2020-0233

SWIFT

2020-0173

2020-0250

2020-0252

2020-0253

2020-0269

2020-0270

2020-0271
Silicon and Germanium Delta-Doped Diamond Homoepitaxial Films

Low-Melting Oxide-Glass: A New Type of Solid-State Electrolytes

Advanced Materials for Separations Technology via Electrospinning

Scalable Bayesian Calibration for Risk-Conscious Building Energy Modeling

Evaluation of Precipitation Instrument Performance Across Rain Rates

High-Energy X-ray Diffraction and Machine Learning

Driven Molecular Dynamics Simulation Study of Molten Chloride Salts

Developing Cathodes with Advanced Surface

Treatment Towards Future Electron Sources

Advanced Algorithms for Optimizing Critical

Infrastructure Systems Affected by High-Impact Rare

Events

Interlayer Films for Solid State Batteries

High-Fidelity Multiphase Simulation of Steam

Separators in a Light Water Reactor

Slurry Based Flame Spray Pyrolysis for Catalyst

Synthesis and Production

Adaptive-Scale Multivariate Semi-Analytical

Computation Approach for Transient Simulation of

Networked Energy Systems in National Resilience

Analysis

Adsorption Strategies for PFAS

Molecular Engineering of Field-effect Transistor

Sensor for Ultrasensitive Detection of Per- and

Polyfluoroalkyl Substances (PFAS) in Water

Interoperability and Cybersecurity Assessment of

Smart Charge Adapter
Moinuddin Ahmed

35

Matthew Menickelly

35

William Pringle

36

Sudharshan Ashwin

36

Renganathan

Pinaki Pal

37

Nazar Delegan

37

Yujia Liang

38

Lauren Valentino

38

Qi Li

39

Adam K, Theisen

39

Jicheng Guo

40

Jiahang Shao

40

Anirudh Subramanyam

41

Jeffrey W. Elam

42

Lander Ibarra

42

Rongyue Wang

43

Rui Yao

43

Edward F. Barry

44

Junhong Chen

44

Daniel S. Dobrzynski 

with Highly Reusable, Recyclable and Reproducible Properties

2020-0281 Surrogate Fallout for Rad-Nuke Resuspension and Decontamination Studies

Michael D. Kaminski $\quad 46$

Two-Dimensional Numerical Modeling of Tailings Dam Mustafa Siddik Altinakar Failures

Incorporating Artificial Intelligence Capabilities to
Support Critical Infrastructure Interdependency

Lawrence P. Lewis

Analysis

2020-0290

Identify and Catalog Information Sources for Global

Duane R. Verner Energy Security Database

2020-0291 Millifluidic Optical Cell to Measure Uranium

Cari A. Launiere $\quad 48$

$2020-0298$ Enhancing Capabilities of X-ray Diagnostics for

Brandon A. Sforzo Aerospace Propulsion

Dichroic Ptychography for Mapping of Magnetic

Joerg Strempfer

50

2020-0303 Domains and Orbital Anisotropies

Artificial Intelligence Driven Development of a

James J. Davis Compact Antimicrobial Resistance Prediction Platform Using the Cloud Lab

2020-0304 Develop an Agent-Based Model Applicable for Simulating COVID-19 Disease Propagation and Interventions in an Urban Area 


\section{ADVANCED COMPUTING}

\begin{tabular}{|l|l|}
\hline LDRD \# 2018-058 & Advanced Computing $\$ 1,114,940$ \\
\hline Project Title: & $\begin{array}{l}\text { Modeling and Quantifying Tradeoffs among Execution Time, Power, and } \\
\text { Resilience of Parallel Application to Efficiently Exploit System Heterogeneity }\end{array}$ \\
\hline Investigators: & $\begin{array}{l}\text { Valerie Taylor } \\
\text { Xingfu Wu }\end{array}$ \\
\hline
\end{tabular}

\section{Abstract}

As the complexity of high-performance computing (HPC) systems increases, applications face a challenge in achieving energy efficient execution. We conducted extensive experiments on a suite of representative applications and developed effective methods for modeling and quantifying the tradeoffs among execution time, power, and resilience of parallel applications. We used parallel systems with different architectures or accelerators such as the Argonne Leadership Computing Facility (ALCF) Theta and Oak Ridge Leadership Computing Facility (OLCF) Summit. Our modeling research provided a deeper understanding of the interplay for trade-offs among the relevant parameters. We used machine learning to develop hardware counter-based analytical models to quantify the correlations and tradeoffs. Insights from the counters provided guidance on application optimizations to perform informed application modifications, system tuning, or a combination of both. We improved application performance and energy efficiency using those models. For example, we analyzed and improved performance, energy utilization and scalability of parallel CANDLE (CANcer Distributed Learning Environment) benchmarks, on ALCF Theta and OLCF Summit. On Theta, we achieved over $41 \%$ in energy saving under strong scaling on up to 384 nodes. On the heterogenous system Summit, we improved the energy savings by up to $78 \%$ under strong scaling on up to 384 graphical processing units (GPUs) and by up to $77 \%$ under weak scaling on up to 3,072 GPUs. This project has advanced the scientific understanding of the interdependence among power, execution time, and resilience for various application-system configurations. It will aid in the design of parallel applications, runtime systems, and computer architectures going forward.

\begin{tabular}{|l|l|}
\hline LDRD \# 2019-0030 & Advanced Computing \$590,548 \\
\hline Project Title: & Probabilistic Forecasting of Extreme Weather Using Deep Learning \\
\hline Investigators: & $\begin{array}{l}\text { Carlo J. Graziani } \\
\text { Jiali Wang, Veerabhadra R. Kotamarthi }\end{array}$ \\
\hline
\end{tabular}

\footnotetext{
Abstract

Decision-making under uncertainty about the future is a familiar and important activity attending a range of domains including extreme weather. A crucial tool for decision-making under uncertainty is the probabilistic forecast, a tool needed for attaching meaningful probabilities to possible future outcomes enabling quantitative risk assessment and mitigation. Our project uses deep learning techniques to produce probabilistic forecasts of weather. We have demonstrated the viability of our approach to
} 
uncertainty-quantified weather forecasting using the Weather Research and Forecasting (WRF) Model, a next-generation mesoscale numerical weather prediction system designed to serve both atmospheric research and operational forecasting needs. It features two dynamical cores, a data assimilation system, and a software architecture facilitating parallel computation and system extensibility. We used WRF simulations to train a deep forecasting machine to predict wind at a location in Kansas City. This demonstrated that the technique is practical for probabilistic prediction of wind strength and direction. We have also demonstrated that a Jacobian Tracker Neural Network (JTNN) is capable of learning the distribution of weather conditions and can be used to sample the learned distribution to furnish probabilistic forecasts. Lastly, we have translated the Storm Tracker software package from Matlab to Python and are exploring its properties as a feature extraction/dimensional reduction system. Our developed framework addresses both the issue of resilience of the energy distribution grid to extreme weather and the forecast uncertainty problems of important sources of non-hydro renewable energy, i.e., wind and solar.

\begin{tabular}{|l|l|}
\hline LDRD \# 2019-0202 & Advanced Computing \\
\hline Project Title: & $\begin{array}{l}\text { Development and Application of Advanced Computing Technologies and } \\
\text { Models for Hypersonic Combustion Applications }\end{array}$ \\
\hline Investigators: & $\begin{array}{l}\text { Sibendu Som } \\
\text { Prithwish Kundu, Roberto Paoli }\end{array}$ \\
\hline
\end{tabular}

\begin{abstract}
Our goal has been the development of predictive simulation capabilities that can not only be used by the hypersonics community, but also by the gas turbine, detonation, and piston engine communities. We have demonstrated significant capabilities in modeling supersonic combustion in scramjets relevant to high-speed propulsion applications. We have also carried out high fidelity chemical kinetics-based analysis of the HiFIRE (Hypersonic International Flight Research Experimentation Program) scramjet combustor using the CONVERGE code and adaptive meshing. These results were used to investigate the combustion regimes of flamelet models for scramjet configurations. We acquired NASA's VULCANcomputational fluid dynamics (CFD) code, a compressible, turbulent, Navier-Stokes flow solver, and are testing it on Argonne's LCRC clusters as well as on ALCF-Theta. Simulations of the HiFIRE combustor with 7.6 million cells were carried out using the VULCAN code. We have developed a framework to incorporate the transport equations and latest flamelet models into the code. This will enable us to run high fidelity large eddy simulation (HiFiLES) calculations with upwards of 40 million cells. We have finished the implementation of transport equations for mixture fraction variance and progress variable for supersonic combustion in the VULCAN code. The source term calculations for the progress variable have been coupled with the transport equations and 5D interpolation. Finally, we have finished the implementation of the unsteady flamelet progress variable (UFPV) model for supersonic combustion and have tested the code.
\end{abstract}




\begin{tabular}{|l|l|}
\hline LDRD \# 2019-0300 & Advanced Computing $\$ 422,036$ \\
\hline Project Title: & Enabling Automatic Learning of Atmospheric Particles through APS-U \\
\hline Investigators: & $\begin{array}{l}\text { Zichao Di } \\
\text { Yan Feng, Barry Lai }\end{array}$ \\
\hline
\end{tabular}

\title{
Abstract
}

There is a need for new robust data analytics toolkits. We have developed an automated and robust data analysis toolkit for data collected via synchrotron-based X-ray fluorescence coupled with X-ray absorption near edge structure as applied to atmospheric particle samples. To facilitate the analysis, we provide a natural and amendable framework to incorporate a priori training knowledge into automatic extraction through unsupervised multichannel learning. We applied statistical models including clustering and supervised machine learning to processed image datasets in order to provide insights into evolution and chemical transformation of atmospheric particles on both individual and population (or cluster) bases. We addressed a morphology-based image analysis method for particle identification, perparticle-based extraction of elemental concentration, and a data fusion framework of multi-elemental Xray fluorescence images. Our integration of advanced mathematical morphology and unsupervised learning to automate and accelerate Advance Photon Source (APS) extreme data flow and analysis of atmospheric particle images significantly reduces analysis time and human bias compared to the current manual approach. Our method provides a detailed, statistical characterization of observed atmospheric particles by combining multiple-element $x$-ray fluorescence maps. Results can provide critical information about chemical composition, size distribution, and origins of these particles, which play an important role in the Earth's energy balance, cloud formation, and global biogeochemical cycles.

\begin{tabular}{|l|l|}
\hline LDRD \# 2019-0313 & Advanced Computing \\
\hline Project Title: & Neuromorphic Learning for Neuroimaging \\
\hline Investigators: & $\begin{array}{l}\text { Fangfang Xia } \\
\text { Mark Hereld, Neil J. Getty, Thomas S. Brettin }\end{array}$ \\
\hline
\end{tabular}

\begin{abstract}
Our goal has been to develop a new brain-inspired algorithm that is more efficient at learning from data. We made progress on the two main research areas: brain image prediction and neuromorphic learning. We pursued an active low-data learning algorithm implemented on an Intel neuromorphic platform. We demonstrated a working spiking neural network (SNN) model on the Intel Loihi neuromorphic hardware for brain image classification. We explored representative lines of research and demonstrated the utility of our methods on a classification benchmark of brain cancer MRI data. First, we presented a capsule network that explicitly learns a representation robust to rotation and affine transformation. This model requires less training data and outperforms both the original convolution baseline and a previous capsule network implementation. A low-data learning algorithm can benefit many scientific domains for which labeled data are not abundant. Second, we leveraged the latest domain adaptation techniques to achieve a new state-of-the-art accuracy. Our experiments showed that non-medical images can be used to improve model performance. Third, we designed a SNN trained on the Intel Loihi neuromorphic chip.
\end{abstract}


This model consumes much lower power while achieving reasonable accuracy given model reduction. We explored data efficient learning methods for images that deviate from conventional deep learning. Our implementation utilized learning rate decay, larger batch sizes and longer training times.

\begin{tabular}{|l|l|}
\hline LDRD \# 2020-0473 & Advanced Computing $\quad \$ 474,162$ \\
\hline Project Title: & Advanced Computing Expedition 2020 \\
\hline Investigators: & Michael Papka \\
\hline
\end{tabular}

\section{Abstract}

Our fast-track focused efforts are on gaining a better understanding of cutting-edge computational components in support of science. Our work enables quick, low-cost, and potentially high-reward prototyping efforts. Ten campaigns within our project all targeted artificial intelligence/machine learning problem areas as enumerated here:

- The inverse problem in rotational spectroscopy

- Expanding Deep Learning Network Anomaly Detection

- Al for Thermochemistry

- Improvements to cosmic muon identification at Muon-to-Electron Conversion Experiment (Mu2e)

- Neural Network Potentials for Simple Alkanes

- Deep learning speed up high-resolution climate simulations

- Self-supervised deep learning for x-ray imaging without reference data

- The Use of Metabarcoding to characterize Ecosystem Health Status

- An Active Learning Workflow for Machine Learning Forcefield Automation

- Predict PFAS (per- and polyfluoroalkyl substances) toxicity with a deep learning approach

Exploration of these innovative approaches to new computing technologies will result in a collection of reports, code repositories, and slide presentations upon which further extensive pursuits can be based. 


\begin{tabular}{|l|l|}
\hline LDRD \# 2020-0168 & Al + Science \\
\hline Project Title: & Al Robotics for Solution-Processable Materials \\
\hline Investigators: & $\begin{array}{l}\text { Pierre T. Darancet } \\
\text { Subramanian Sankaranarayanan, Logan T. Ward, Angel Yanguas-Gil, Jie Xu, } \\
\text { Young Soo Park }\end{array}$ \\
\hline
\end{tabular}

\begin{abstract}
The need for optimized solution processing conditions is ubiquitous in emerging technologies, impacting fields as diverse as electronics, solar energy conversion and multifunctional coatings. However, obtaining the target material with desired properties through solution-processing is time intensive because the number of available processing parameters is quite large. This project accelerates the development of synthesis of complex nanomaterials with target properties through the integration of physics-guided artificial intelligence (Al), robotics, and high-throughput synthesis methods. We developed autonomous synthesis loops in which an automated modular robotic system performing both material synthesis and characterization is guided by artificial intelligence ( $\mathrm{Al}$ ) algorithms that efficiently reduce exploration of the synthesis and processing parameter space. We developed the Al component based on experimental synthesis data and on synthetic data from molecular dynamics and tight binding simulations. The first research objective was maximization of morphological ordering in conjugated polymer films through directional solution-coating. These are emerging as promising building blocks for a broad range of applications including flexible electronics, optoelectronics, clean energy and sensing technologies.
\end{abstract}

\begin{tabular}{|l|l|}
\hline LDRD \# 2020-0169 & Al + Science \\
\hline Project Title: & $\begin{array}{l}\text { Al-driven Design of Controlled Phase Separation Mediated by Intrinsically } \\
\text { Disordered Peptide Polymers }\end{array}$ \\
\hline Investigators: & $\begin{array}{l}\text { Arvind Ramanathan } \\
\text { Gyorgy Babnigg, Marie-Francoise Gros }\end{array}$ \\
\hline
\end{tabular}

\begin{abstract}
The primary goal of our project was to use an automated robotic platform, guided by a probabilistic experimental design strategy to design intrinsically disordered peptide polymers (IDPPs) that undergo liquid-liquid phase separation (LLPS) under specific physiological conditions (e.g., temperature, pH, presence of solvents, etc.). We began with developing an automated platform for screening small peptides that could self-assemble into phase separated components. We acquired a Hudson Robotic liquid handling platform and developed a Python code-based open-source application programming interface (API) that could perform many standard liquid handling operations at scale on this robot. We followed a protocol that included robotic assembly of IDPP libraries and purification of fluorescent protein fusions; development of probabilistic models to filter and rank IDPPs that undergo LLPS; validation of automated screening protocols for the IDPP designs; and assembly of libraries of IDPPs
\end{abstract}


with tunable LLPS behavior for targeted applications. The protocols were implemented and tested for screening peptide designs and we have demonstrated its capability in designing a known peptide that undergoes phase separation.

\begin{tabular}{|l|l|}
\hline LDRD \# 2020-0170 & Al + Science \\
\hline Project Title: & $\begin{array}{l}\text { Building an Autonomous Materials Discovery (AMD) System for Metamaterials } \\
\text { and the Associated Data Infrastructure }\end{array}$ \\
\hline Investigators: & $\begin{array}{l}\text { Benjamin J. Blaiszik } \\
\text { Marcus E. Schwarting, Juan J. de Pablo, Ian T. Foster, Young Soo Park }\end{array}$ \\
\hline
\end{tabular}

\begin{abstract}
An autonomous materials discovery (AMD) system couples machine learning (ML) models, simulation, automated material assembly, and automated experimental material characterization in a feedback loop. We pursued exploration of the data infrastructure necessary to support Al-driven automated laboratories, establishing a model autonomous design, experiment, and characterization capability and applying this capability to design challenges in metamaterials. The specific experimental capability provides an actual demonstration of how simulation, experiment, and machine learning can be combined in an automated materials discovery loop. The experimental setup consisted of 3D material printers capable of printing soft materials coupled with programmable robotic arms and characterization equipment to enable automated testing of the printed metamaterials. Our initial target was to autonomously discover material structures that result in varying elastic properties, improved fracture toughness, and 3D auxetic metamaterials. This project linked existing autonomous laboratory efforts and provided a clear analogue for future discovery capabilities, especially those involving 3D printing.
\end{abstract}

\title{
BIOLOGICAL AND ENVIRONMENTAL SCIENCE
}

\begin{tabular}{|l|l|}
\hline LDRD \# 2018-055 & Biological and Environmental Science $\quad \$ 424,942$ \\
\hline Project Title: & $\begin{array}{l}\text { Variability and Trends in Land Surface Properties: Effects on Boundary Layer } \\
\text { Physics and Extremes Onset }\end{array}$ \\
\hline Investigators: & $\begin{array}{l}\text { Yan Feng } \\
\text { Veerabhadra R. Kotamarthi, Nicki L. Hickmon, Ryan Sullivan, Virendra P. Ghate }\end{array}$ \\
\hline
\end{tabular}

\section{Abstract}

Extreme weather events are of great importance to national resilience and global security. However, understanding of the drivers and triggers of these events remains limited, preventing improvement of atmospheric model predictability and associated support for decision making. We know, for example, a key mechanism underlying mega-heatwaves is the coupling of soil moisture, surface heat and moisture fluxes, and air temperature. Surface flux and moisture are highly variable in time and space at the air- 
land interface, thus presenting a challenge in measurements for extreme event detection. We have created a high-quality, spatially and temporally continuous dataset of surface fluxes and soil moisture over the US. Data was obtained from the Atmospheric Radiation Measurement (ARM) user facility's Southern Great Plains (SGP) atmospheric observatory as well as from other sources. Neural networking (NN) algorithms were used to fill data gaps in time and space. We further developed a methodology using an observational database to obtain improved evapotranspiration flux estimates at higher spatial and temporal resolutions, relative to the standard satellite retrievals. The new datasets of surface fluxes and soil moisture have also been used to evaluate the results of the Coupled Model Intercomparison Project climate models (CMIP5 and CMIP6). An event catalogue of extreme event onsets from meteorological observations along with identification of the event onsets that could be predicted based on surface flux datasets using known physical mechanisms informs simulations with high-resolution mesoscale models. The data from observations and models are used to build a conceptual model of event triggers. Our findings have important implications for improving predictability of climate models and modeling and projecting extreme weather events.

\begin{tabular}{|l|l|}
\hline LDRD \# 2019-0340 & Biological and Environmental Science \$1,224,924 \\
\hline Project Title: & $\begin{array}{l}\text { Design and Test Synthetic Pathways to Optimize Their Effectiveness and } \\
\text { Produce New Chemical Diversity }\end{array}$ \\
\hline Investigators: & $\begin{array}{l}\text { Michael Fonstein } \\
\text { Ross Overbeek, Gyorgy Babnigg, Christopher S. Henry }\end{array}$ \\
\hline
\end{tabular}

\section{Abstract}

Our project represents an expansion of synthetic biology approaches. We created a combinatorial biochemistry system capable of functioning similarly to combinatorial chemistry systems that are often used in the pharmaceutical industry today. We developed a system of carefully selected promiscuous enzymes, cofactors, and starting backbone molecules that, when mixed, rapidly produce a wide range of output molecules and derivatives by enzymatically performing combinatorial chemistry on a starting backbone molecule. We have completed several key parts of our research plan, including the testing and optimization of systematic deletion technology for host construction. In addition, we constructed multiple engineered bacterial strains for the hosting of initial synthetic constructs. Specifically, a targeted deletion technology for host construction was optimized (cycle time cut from two weeks to three days); multiple bacterial strains for hosting of the synthetic gene modules were constructed (32 host strains carrying combinations of two to four various deletions of twelve different genes); and multiple gene modules were constructed and put under optogenetic and IPTG (Isopropyl $\beta$-d-1thiogalactopyranoside) controls (nine core regulated threonine-producing modules and ten plasmid constructs carrying supplementary genes). In addition, four different threonine modules and ten supplementary single gene constructs were introduced into 32 complex deletion hosts; the relative expression rates for genes which were used in construction and are important for backbone biosynthesis were established based on whole-genome ribonucleic acid (RNA) sequencing; and optogenetic regulators were redesigned and adapted to fit our experimental design. A device capable of providing regulated light of various wavelengths to cells growing in a 96-well format was also constructed. Threonine production data were collected and subjected to computational analysis and the 
first twelve "production" samples were subjected to whole-genome RNA sequencing. Finally, a computational environment was constructed to support curation and artificial intelligence (AI) analysis of high-throughput screening (HTS) data.

\title{
ENERGY STORAGE
}

\begin{tabular}{|l|l|}
\hline LDRD \# 2018-100 & Energy Storage \\
\hline Project Title: & $\begin{array}{l}\text { High-Density Energy Storage in Solvent-Free Redox Flow Batteries for Flexible } \\
\text { Electric Grid }\end{array}$ \\
\hline Investigators: & $\begin{array}{l}\text { Lu Zhang } \\
\text { llya A. Shkrob }\end{array}$ \\
\hline
\end{tabular}

\begin{abstract}
A continuing challenge for the electric grid is uneven demand. By adding large-capacity energy storage units to the grid, power generated during quiet times and/or from renewable power resources can be stored and used when demand for power is high. Efficient and economical large-capacity energy storage systems are essential. Redox flow battery (RFBs) systems that enable large scale energy storage present an opportunity to address this need. Among RFB chemistries, hybrid nonaqueous RFBs (HNRFBs) stand out by providing the lowest cost due to high theoretical energy density. Our goal was to develop solvent-lean HNRFBs with a volumetric energy density greater than 200 Watt-hours/liter (Wh/L), which is about eight times greater than in current aqueous RFBs and two to three times greater than in current nonaqueous RFBs. The key innovation is in developing liquid redox materials for RFBs that comprise a maximum possible fraction of the electrolyte. Two families of liquid catholyte molecules (i.e., redox active organic molecules (ROM)) with theoretical volumetric energy density over 200(Wh/L) were developed. We also developed a hybrid cell that can couple catholytes directly with a lithium anode. We extended our effort to develop novel liquid catholyte materials, particularly two-electron liquid cahtolyte molecules that hold promise to remedy viscosity and conductivity issues of the high concentration cathloytes. We are using small angle $x$-ray spectroscopy (SAXS) and density functional theory (DFT) to correlate aggregates and solvation energies to structural changes, which when combined with computation, may lead to understanding solvation behavior of such catholytes. While there are many technical barriers still existing for high concentration catholytes, such as the increases of viscosity, reduction of conductivity, etc., our work has begun to approach development of a hybrid cell with the target energy density over $200 \mathrm{Wh} / \mathrm{L}$.
\end{abstract}




\begin{tabular}{|l|l|}
\hline LDRD \# 2018-104 & Energy Storage \\
\hline Project Title: & Complex Fluids from Chemical Separations: A New Entry to Battery Electrolytes \\
\hline Investigators: & $\begin{array}{l}\text { Lynda Soderholm } \\
\text { Mark Antonio, Wei Jiang }\end{array}$ \\
\hline
\end{tabular}

\begin{abstract}
We sought a new perspective on researching electrolytes for high-voltage applications. Our goals were to transfer knowledge from solvent extraction (SX) solution studies to facilitate design-to-synthesis of hierarchically structured, highly loaded electrolytes; to use characterization approaches and tools from SX studies to link solution correlations with influences on solute-transport behavior in electrolytes as a function of applied voltage; and to seek local and longer-range structural attributes that allow facile and selective incorporation of the lithium ion $\left(\mathrm{Li}^{+}\right)$charge carrier while preventing dissolution of cathodic transition-metal components ( $\mathrm{Mn}, \mathrm{Co}, \mathrm{Ni}$ ions). Underpinning our approach is the application of lessons learned from third-phase formation in liquid-liquid extraction (LLE) systems. LLE involves the selective ion transfer from a high-dielectric solution to an immiscible non-polar liquid into which an amphiphile has been dissolved. We pursued a scoping study designed to delineate the constituents and structures of complex fluids that may have application as electrolytes in high-performance batteries. The idea was to synthesize fluids with laminar structures that can act as low-resistance media for $\mathrm{Li}^{+}$transport between electrodes. Our initial studies were based closely on conventional LLE systems, using a variety of molecular amphiphiles. Then, we moved to amphiphile- and acid-types that have seen use in battery applications and we investigated replacing water as the high-dielectric solvent with something more robust to the application of high voltage. Having synthesized a variety of third-phase, structured fluids, we tested some of them for their ionic conductivity over a broad potential window. We were able to demonstrate that several of the fluids had wide stability windows (in excess of $3 \mathrm{~V}$ ) but their resistance and conductivities were too high. Finally, small-angle X-ray scattering (SAXS) data have been obtained, the analysis of which will link solution structure with the conductivities.
\end{abstract}

\begin{tabular}{|l|l|}
\hline LDRD \# 2018-105 & Energy Storage $\quad$ \$290,888 \\
\hline Project Title: & Electrode Contamination Investigation to Advance Direct Recycling \\
\hline Investigators: & $\begin{array}{l}\text { Jeffrey Spangenberger } \\
\text { Bryant J. Polzin }\end{array}$ \\
\hline
\end{tabular}

\begin{abstract}
Direct recycling of lithium-ion batteries has great potential to make the process profitable, however, there are many challenges to overcome. Today's direct recycling research is done at bench scale with researchers dismantling batteries by hand to recover the active electrode. That results in very clean starting material but is cost prohibitive at large scale. Batteries need to be shredded to liberate the electrode powders at scale. Several companies generate what is known as "black mass" at various scales. They typically send their black mass for pyrometallurgical or hydrometallurgical treatment. It is known that contamination in the recovered material causes reduced cell performance or even battery cell failure. We analyzed and identified key causes of reduced performance and cell failure in order to
\end{abstract}


determine how best to mitigate this issue. Our focus was on discovering how contaminants in recovered cathode powders can affect the performance of new batteries with the recycled cathode material. We characterized several examples of battery materials collected from current recyclers to identify contaminants. These contaminants were then studied individually when cast into new batteries and cycled, to determine their effect on performance. Those results were then compared to performance with cells fabricated using pristine materials. We also evaluated processing equipment that can reduce or remove contaminants in order to reduce separation/purification costs downstream. The findings of our study determined which contaminants cause reduced cell performance or failure and indicated whether the failure was due to anode, cathode, or both electrodes.

\section{HARD X-RAY SCIENCES}

\begin{tabular}{|l|l|}
\hline LDRD \# 2018-002 & Hard X-ray Sciences \\
\hline Project Title: & $\begin{array}{l}\text { Development of a Hard X-ray Spectrometer Based on Transition Edge Sensors } \\
\text { for Advanced Spectroscopy }\end{array}$ \\
\hline Investigators: & $\begin{array}{l}\text { Orlando Quaranta } \\
\text { Tejas Guruswamy, Umeshkumar Manibhai Patel }\end{array}$ \\
\hline
\end{tabular}

\section{Abstract}

We sought to devise a new $\mathrm{x}$-ray detector that allows application of techniques in spectroscopy not previously possible and that would be a unique capability at Argonne's Advance Photon Source (APS). The design and fabrication of the instrument utilizes a transition edge sensor (TES) array along with its cryogenic system. These sensors have energy resolutions one to two orders of magnitude better than semiconductor alternatives. The design comprises about 100 pixels with a saturation energy of about $20 \mathrm{keV}$, energy resolution of about $10 \mathrm{eV}$, and is able to handle count rates of 10 to 100 counts/second/pixel. Several initial wafers were produced to test fabrication, and a complete set of working devices was produced. Software and hardware were received from the National Institute of Standards and Technology (NIST) and a cryostat for the operation of the sensors was acquired. Substantial development effort was devoted to the sensors, cryogenics and readout electronics. Two versions of a second set of devices were fabricated with saturation energies of about $8 \mathrm{keV}$ and $25 \mathrm{keV}$, and energy resolutions of about $8 \mathrm{eV}$ and $11 \mathrm{eV}$, respectively. The new detector setup was moved into the beamline 1-BM-C hutch at APS. Development of large sensor arrays to increase throughput was pursued while running $x$-ray spectroscopy experiments with limited size arrays. The instrument has been used to both test sensor design in the beamline environment and to run representative experiments. For example, fluorescence experiments were run to map elemental composition with detail that wasn't previously reachable. The instrument is now completely deployed at 1-BM-C and in routine use. 


\begin{tabular}{|l|l|}
\hline LDRD \# 2018-016 & Hard X-ray Sciences \\
\hline Project Title: & $\begin{array}{l}\text { Development of Zoom Optics Using Deformable Mirrors with in situ Wavefront } \\
\text { Sensing and a Closed-loop Control System }\end{array}$ \\
\hline Investigators: & $\begin{array}{l}\text { Lahsen Assoufid } \\
\text { Ross J. Harder, Deming Shu, Xianbo Shi }\end{array}$ \\
\hline
\end{tabular}

\title{
Abstract
}

This project aimed to develop technology for mirror-based zoom x-ray optics, which will offer variable two-dimensional (2-D) focal spot size, at the experiment sample location, over a wide range - from a few tens of nanometers to micrometers with long-duration stability. Continuously deformable mirrors offer a tremendous advantage, not only in terms of accuracy, reliability, and flexibility, but also in producing the optimal $x$-ray spot size. A significant and critical part of this project is the development and integration of wavefront measurements and control in a closed-loop system, plus related software and algorithms for automatic mirror adjustment and focus control. We demonstrated the concept using a one-dimensional (1-D) version, consisting of two sequential mirrors incorporating in situ metrology and an at-wavelength wavefront sensor for beam wavefront measurement and feedback control. The first/upstream mirror was an in-house-developed mirror with a flexure-based mechanical bender providing an intermediate focus. The second/downstream mirror was a commercial bimorph deformable mirror designed to generate a predetermined spot size. An optic of this type is essential for coherent nanoprobe beamlines and many other beamlines for coherent imaging and x-ray microscopy. Initially, we developed the basic transfer function and control algorithm of a deformable mirror using an existing, low-resolution piezoelectric lead zirconate titanate (PZT)-based bimorph mirror and a grating interferometer as a wavefront sensor. The capacitive-sensor-based mirror surface shape measurement and monitoring were thoroughly tested and calibrated by the optical metrology and X-ray wavefront sensing technique. We evaluated our prototype using the Advanced Photon Source (APS) bending magnet beamline, 1-BM, demonstrating its ability to continuously adjust the beam focal spot size over a wide range of sizes. Additionally, a machine-learning ( $\mathrm{ML}$ ) control algorithm was developed for the upstream bender mirror. The knowledge acquired in this area later served as the basis to create zoom mirror optics for APS future beamlines.

\begin{tabular}{|l|l|}
\hline LDRD \# 2018-019 & Hard X-ray Sciences \\
\hline Project Title: & A.I C.D.I: Atomistically Informed Coherent Diffraction Imaging \\
\hline Investigators: & $\begin{array}{l}\text { Mathew Cherukara } \\
\text { Ross J. Harder, Subramanian Sankaranarayanan, Youssef Nashed }\end{array}$ \\
\hline
\end{tabular}

\begin{abstract}
Coherent X-ray diffraction imaging (CDI) is a powerful technique for operando materials characterization at the nanoscale. Coherent imaging techniques are the primary driver for upgrades to several synchrotron sources including the Advanced Photon Source (APS). The method's main challenge is recovery of the phases of scattered $x$-rays which are needed to extract an image of the sample from the data. The phase retrieval process can be computationally expensive, and the current methods have
\end{abstract}


inherent deficiencies. They do not include physical knowledge of the material or the phenomena being studied, and the resulting image is limited by the signal-to-noise ratio in the data. Both the resolution and knowledge acquired from CDI may improve through data analysis methods that incorporate the physics of the material and process being studied. Our approach is to replace the phase retrieval step in $\mathrm{CDI}$ with machine learning (ML) algorithms that, once trained, predict an object's structure and phase in a single step based on a training database derived from large scale molecular dynamics (MD) and statics (MS) simulations. The fitted image is inherently physical since it is derived from the true physical properties of the atoms making up the material, i.e., it is atomistically informed CDI (AICDI). We developed the first end-to-end deep neural network solution (CDI-NN) to the phase-retrieval problem in the far-field. The CDI-NN as compared to traditional phase retrieval is 500 times faster, works more effectively with strong phase objects, and requires no user input. We used an atomistic model to explain observed phases in a CDI image reconstruction and the data were also a test bed for neural network identification of defects. Both simulation data and manually tagged real-data from the APS were used for validating the CDI-NN and for calibrating the model. This was an iterative process where the data from MS simulations are used systematically for reconfiguring the CDI-NN layers and networks to improve the accuracy on the manually tagged real-data. Our approach extracts an unprecedented level of clarity and conciseness from measured data. It gives researchers the ability to refine their experiment with intelligence provided by concurrently running simulations that couple to experimentally observed structures in real time.

\begin{tabular}{|l|l|}
\hline LDRD \# 2018-027 & Hard X-ray Sciences $\quad \$ 649,788$ \\
\hline Project Title: & $\begin{array}{l}\text { Dark-Field Imaging of Multiscale Structures and Their Role at Phase Transition, } \\
\text { Hysteresis, and Relaxation, Tuned by Magnetic Field and Temperature }\end{array}$ \\
\hline Investigators: & $\begin{array}{l}\text { Zahirul Islam } \\
\text { Lahsen Assoufid }\end{array}$ \\
\hline
\end{tabular}

\footnotetext{
Abstract

The dark-field $\mathrm{x}$-ray microscope (DFXM) will expand the imaging portfolio and exploit the increased beam coherence at the Advanced Photon Source Upgrade (APS-U). The use of nano-calorimetry and a trapped flux magnet (TFM) eliminates external perturbations of sample position and orientation on field-temperature changes of commercial cryostats. Advanced sample environments make such a facility uniquely attractive to researchers to visualize phase transitions as a function of field and temperature. Our project will result in the development of a high-resolution, diffraction-based, bulk-sensitive microscope, applicable to a wide range of materials. Key components of a DFXM include illumination optics, an objective lens (OL) after the sample, a scintillator, and optics for recording a visible-light image to a CCD camera. Prototype of a mechanical manipulator for objective lens (OL): A novel mechanical design for a precision alignment apparatus for a compact $x$-ray compound refractive lens $(C R L)$ manipulator was developed. Commonly, control of $x$ - ray focal distance requires bulky vacuum chambers with manipulators that are permanently deployed as a beamline optical element. This is not suitable on high-resolution diffractometer detector arms required for dark-field x-ray microscopy (DFXM) studies. They require a radically different light-weight design. The novelty of our new mechanical design is its compactness and positioning stability as well as repeatability of its unique
} 
flexural lens holder alignment structure. A prototype has been built and tested. Characterization of Be lenses: Fifty two-dimensional parabolic beryllium (Be) lenses were obtained and characterized at the APS 1-BM beamline. The thickness profile of each lens was measured with the grating interferometry. For precision alignment, the lenses were assembled using the aforementioned manipulator. The repeatability of the manipulator was thoroughly tested. Polymeric Lenses: Also, a pair of polymer-based $\mathrm{x}$-ray prism lenses and a compound refractive lens fabricated at Karlsruhe Institute of Technology were characterized on an insertion device beamline at the APS. Proof-of-principle DFXM experiment: We conducted experiments with an imaging setup using a zone-plate objective similar to the proposed DFXM. We studied contrast mechanisms and tested geometric precision of data collection. Our goal of a controlled scheme of bringing in an $\mathrm{OL}$, changing its focal length and magnification on demand, to image sample transformations under real conditions has been achieved.

\begin{tabular}{|l|l|}
\hline LDRD \# 2018-028 & Hard X-ray Sciences \\
\hline Project Title: & $\begin{array}{l}\text { Integrated Approach to Unravel Four Dimensional Spatiotemporal Correlation } \\
\text { in Highly Transient Phenomena: Ultrafast X-ray Imaging and High-Performance } \\
\text { Computing }\end{array}$ \\
\hline Investigators: & $\begin{array}{l}\text { Jin Wang } \\
\text { Zhang Jiang, Francesco De Carlo, Tao Sun, Nicola J. Ferrier, Wei Jiang }\end{array}$ \\
\hline
\end{tabular}

\footnotetext{
Abstract

The ultimate result of our work is a suite of experimental and computational tools that benefit the current imaging and scattering community and also lay the foundation for high-resolution 4D imaging and coherent scattering. The method takes advantage of the intense, coherent, high-repetition-rate, $x$ ray pulses of an upgraded Advanced Photon Source (APS). Our concept rests on combining ultrafast $x-$ ray imaging and high-performance computing (HPC) to understand spatiotemporal correlations. Our project entails three confirmed hypotheses. (1) Serial microtomography is feasible and provides a means of understanding heterogeneous and dynamic systems using ultrafast $x$-ray imaging and scattering. A test of this hypothesis results in a time-resolved "accurate" tomographic structure of the single particles. (2) A 3D structure can be reconstructed with 2D images of a finite number of real-space structures whose $2 \mathrm{D}$ projection can be calculated by using physics-based imaging/scattering contrast models. We facilitated the reconstruction of a 3D structure from time-resolved 2D images by generating a set of physical objects like droplets, ligaments, and sheets, projecting the 3D structure to a 2D pattern using phase-contrast imaging theory in different orientations, and with high-fidelity pattern and feature matching, performed machine learning to assign the patterns to 3D structures. (3) Single-pulse images can be recovered or extracted from motion-blurred x-ray images taken with multiple-pulse exposures. This required that we solve the velocity field and transforming object shapes from the overlapped but perfectly sharp images. Our computational approach mimics the process for "blurred" x-ray images. We optimized ultrafast imaging fluid morphology and dynamics using the APS x-ray beam. More importantly, we pioneered the scattering theory and artificial intelligence (AI)-based algorithm that simulate $x$-ray images that yield various liquid morphologies involved in the liquid breakup processes and cavitating flows. The computational method can be used to simulate other ultrafast phenomena
} 
such as additive manufacturing. We also developed high-fidelity computational fluid dynamics simulation as part of the imaging recognition and structure reconstruction.

\begin{tabular}{|l|l|}
\hline LDRD \# 2018-030 & Hard X-ray Sciences \\
\hline Project Title: & $\begin{array}{l}\text { Novel Capabilities for Ultra-fast and Ultra-low-dose 3D Scanning Hard X-ray } \\
\text { Microscopy }\end{array}$ \\
\hline Investigators: & $\begin{array}{l}\text { Doga Gursoy } \\
\text { Sunil Bean, Tekin Bicer, Sven Leyffer, Junjing Deng, Si Chen, Sven Leyffer, } \\
\text { William R. Scullin }\end{array}$ \\
\hline
\end{tabular}

\begin{abstract}
Our project aimed to explore and develop new capabilities for high-speed and/or low-dose threedimensional (3D) imaging of samples using the scanning x-ray microscopy systems at Argonne's Advanced Photon Source (APS). We intended to provide capabilities vital to the design and operation of future hard $\mathrm{x}$-ray microscope (HXM) beamlines by initiating computational methods that couple experiment design and analysis. Our approach is based on co-design of the instrument with algorithmic and computational methods to achieve improvements in spatial and time resolution of the microscopes. We made substantial progress toward development of (1) new analysis methods for ultra-fast ptychography, (2) multislice methods for imaging thick objects, (3) high-performance computing (HPC) capabilities, and (4) new instrument control systems integrated with analysis and computing methods that enable ultra-fast experimentation. In particular, we generalized optimization techniques for unconventional data collection, developed prototype parallel reconstruction software and control \& metrology techniques for trajectory scanning, and finalized the design of the sample staging and interferometry stage for measuring sample position in three dimensions (3D). We also developed computational imaging approaches and Bayesian models, multi-slice methods for imaging thick objects, and design of sample-scanning instrumentation and the metrology setup. The Bayesian framework was introduced for ptycho-tomography under photon-limited conditions. Plus, we introduced automatic differentiation for $x$-ray nanotomography beyond the depth of focus limit. To analyze the large amount of data with the new methods and to obtain fast feedback at the beamline, we developed a streamingcapable high-throughput analysis pipeline between the APS and Argonne's Leadership Computing Facility (ALCF) and demonstrated production-ready ptycho-tomography reconstruction software on ALCF's supercomputers. Finally, we demonstrated the trajectory scanning and analysis capabilities on real data.
\end{abstract}




\begin{tabular}{|l|l|}
\hline LDRD \# 2018-054 & Hard X-ray Sciences \\
\hline Project Title: & $\begin{array}{l}\text { Development of a Compact 352-MHz High Efficiency Solid State RF Amplifier } \\
\text { System Utilizing Enhanced Digital Control }\end{array}$ \\
\hline Investigators: & $\begin{array}{l}\text { Alireza Nassiri } \\
\text { Geoff Waldschmidt, Timothy J. Madden, Aditya Goel, David J. Bromberek, } \\
\text { Douglas Horan }\end{array}$ \\
\hline
\end{tabular}

\begin{abstract}
Our aim was development of a demonstration high-power 352-megahertz ( $\mathrm{MHz}$ ) solid-state radio frequency (RF) power amplifier system using 2-kilowatt (kW) modules and a resonant output combining cavity. The 2-kW amplifier design minimizes size and reduces device temperature. The entire 2-kW RF amplifier chain is developed as a single module that incorporates pre-driver, driver, and final stage power amplifier. Integral microstrip-based directional couplers providing 50-decibel (dB) coupling is also incorporated in the design, thus eliminating the need for discrete units. We completed the design of the new RF combining cavity, including detailed RF, mechanical, and thermal analyses. A panel of experts reviewed the cavity system before the fabrication process commenced. We developed a detailed plan to test the RF combing cavity using both a commercially developed solid-state system as well as using an existing APS high power klystron source. Implementation of the solid-state RF amplifier replacement for the current 1-megawatt (MW) klystrons at the Advanced Photon Source (APS) addresses issues related to vacuum tubes, such as lifetime, complex high-voltage power system, high cost, and reliance on only one non-U.S. worldwide klystron manufacturer.
\end{abstract}

\begin{tabular}{|l|l|}
\hline LDRD \# 2019-0028 & Hard X-ray Sciences \\
\hline Project Title: & Cryogen Free Universal Superconducting Undulator SCAPE \\
\hline Investigators: & $\begin{array}{l}\text { Yury Ivanyushenkov } \\
\text { Ibrahim Kesgin, Matthew T. Kasa }\end{array}$ \\
\hline
\end{tabular}

\begin{abstract}
The Advanced Photon Source Upgrade will allow high-sensitivity dichroic measurements of internal magnetic or chiral structures with ptychographic/tomographic techniques. We aimed to extend the capability of superconducting undulator (SCU) technology to generate, and to provide fast switching between, circularly-polarized photon beams with opposite helicity and between linearly-polarized photon beams of orthogonal linear polarization for high-energy x-rays up to $30 \mathrm{keV}$. We tested the concept of using small current bumps to shift the undulator harmonic energy in either one of two universal SCU devices collocated in a straight section, each preset to a well-defined polarization state. A novel winding scheme for a universal superconducting undulator SCAPE (Super Conducting Arbitrarily Polarizing Emitter) was successfully tested with an undulator model and showed a feasibility of achieving polarization switching with a frequency of up to 10 hertz $(\mathrm{Hz})$. We carried out an experimental and theoretical study of the alternating current (AC) losses in SCAPE coils for which we designed and built a setup (based on a single cryocooler) as well as three SCAPE magnet models. Each model was wound with different superconducting wires, which allowed study of the dependence of AC losses on
\end{abstract}


various parameters of a superconductor. For instance, for one model wound with a "standard" niobiumtitanium conductor, we found that the losses quadratically depend on the AC ripple field amplitude but only linearly on the AC ripple field frequency.

\title{
MANUFACTURING SCIENCE AND ENGINEERING
}

\begin{tabular}{|l|l|}
\hline LDRD \# 2019-0026 & Manufacturing Science and Engineering $\$ 651,382$ \\
\hline Project Title: & $\begin{array}{l}\text { Establishment of an Electrospinning-based Nanofiber Manufacturing Platform } \\
\text { using in situ SAXS/WAXS Measurements }\end{array}$ \\
\hline Investigators: & $\begin{array}{l}\text { Yuepeng Zhang } \\
\text { Hee Je Seong, Erik Dahl, Byeongdu Lee }\end{array}$ \\
\hline
\end{tabular}

\begin{abstract}
Advancement in the scalable synthesis of high-aspect-ratio nanofibers and three-dimensional mesostructures is an advanced manufacturing goal. The development of high-quality polymer, oxide, and metallic nanostructures will enable new materials development for batteries, fuel cells, catalysts, membranes, sensors and communication devices. Our aim was to accelerate electrospinning platform technology development through in situ small angle x-ray scattering (SAXS) study of electrospinning at the Advanced Photon Source (APS). We seek an in-depth understanding of the intermediate and transient phenomena involved in the electrospinning process, along with better knowledge of the correlation between nanofiber material properties and synthesis parameters. With extensive beam experiments at the APS 12-ID-B beamline, we provided a demonstration of high-throughput characterization of 94 electrospinning recipes. This development introduced new synthesis capabilities for one-dimensional (1D) nanomaterial and nonwoven structures at Argonne's Materials Engineering Research Facility (MERF), and it also added nanofiber-based solid-state electrolytes to the Argonne lithium battery material pallet. We subsequently repurposed the electrospinning platform to develop reusable N95 facemask filter media. This activity was part of the National Virtual Biological Laboratory program. We expanded the capability of electrospinning to include advanced membrane development for water filtration and $\mathrm{CO}_{2}$ reduction.
\end{abstract}

\begin{tabular}{|l|l|}
\hline LDRD \# 2019-0254 & Manufacturing Science and Engineering \$606,512 \\
\hline Project Title: & Near Real-Time Optimization of Manufacturing Processes via Machine Learning \\
\hline Investigators: & $\begin{array}{l}\text { Marius Stan } \\
\text { Joseph A. Libera, Elise Jennings, Roberto Torelli, Debolina Dasgupta, } \\
\text { Noah Paulson, Pinaki Pal }\end{array}$ \\
\hline
\end{tabular}

\section{Abstract}

Our goal is to build a human-machine partnership by providing the necessary knowledge, analysis, and computational tools that help to understand and optimize material properties and process parameter 
models during manufacturing. To accomplish that, we pursued advancement of property-process model development, evaluation of model quality, including uncertainty and predictability, and testing the methodology. We chose to perform the testing on the flame spray pyrolysis (FSP) process at Argonne's Materials Engineering Research Facility (MERF). In conjunction with the FSP testing, we formulated a FSP Bayesian optimization loop to optimize resulting particle size distributions and validated a computational fluid dynamics (CFD) model with the data. CFD simulations predict spray atomization, mixing, flame structure, gas-phase chemistry, and particle growth. Machine learning (ML) algorithms probe the large design/process parameter space for optimum baselines. Critical to the success of the near real time optimization procedure was the development of strategies and analyses to efficiently perform experiments and extract robust measurements. Assuring repeatability of measurements, extracting meaningful statistics from the measurements, and assessing the design of experiments were required. Specifically, we explored the relationship between a variety of FSP processing parameters and the resulting particle size distributions. We confirmed the expectation that at low concentrations, FSP results in discrete fundamental particles, while at high concentrations, particles agglomerate in the flame, forming conglomerates of fundamental particles.

\begin{tabular}{|l|l|}
\hline LDRD \# 2019-0279 & Manufacturing Science and Engineering $\$ 638,270$ \\
\hline Project Title: & Develop Spectroscopy and Imaging Capabilities for Microfluidic Channels \\
\hline Investigators: & $\begin{array}{l}\text { Xiao-Min Lin } \\
\text { Jeffrey R. Guest }\end{array}$ \\
\hline
\end{tabular}

\begin{abstract}
A new paradigm of synthesis-on-demand based on microfluidics has emerged in recent decades. To realize its full potential, being able to detect synthesis products in situ is desirable. Our project focused on developing and improving spectroscopy and imaging capabilities to detect chemical reactions in microfluidic channels. We use dark-field scattering coupled with a high-speed camera to track individual particle motion in microfluidic channels. In that way, we derive information such as particle size and shape and their motions. In a driven flow with high particle density, we are implementing nanoparticle image velocimetry to yield valuable information on flow velocity distribution with high spatial resolution. From this we are able to derive experimental parameters that control a complex fluid in a confined space. Coupling this effort with a machine learning $(\mathrm{ML})$ algorithm to control material synthesis in the microfluidic platform is also a possibility. We are constructing a dedicated experimental facility to implement this type of characterization. A particle tracking algorithm has been tested for its applicability and limits in detecting particle motion. A dark-field scattering capability with associated laser systems has also seen substantial progress toward implementation.
\end{abstract}




\begin{tabular}{|l|l|}
\hline LDRD \# 2019-0330 & Manufacturing Science and Engineering $\$ 273,088$ \\
\hline Project Title: & $\begin{array}{l}\text { Integrated Sensor Array and Modeling for Rapid Process Development in } \\
\text { Chemical Vapor Deposition }\end{array}$ \\
\hline Investigators: & $\begin{array}{l}\text { Jeffrey W. Elam } \\
\text { Anil U. Mane, Noah Paulson, Angel Yanguas-Gil }\end{array}$ \\
\hline
\end{tabular}

\title{
Abstract
}

Process development and process optimization are ubiquitous, resource-intensive tasks in thin film research and development. Process development can be accelerated and economized using in situ measurements, but this still requires careful attention of a skilled operator to make informed choices based on experience and intuition. An alternative strategy is use of machine learning $(\mathrm{ML})$ to analyze in situ data in real time and adjust growth conditions based on an algorithm. We developed ML methods to optimize the atomic layer deposition (ALD) of several thin film materials. Initially, we developed the ML code using simulated in situ quartz crystal microbalance (QCM) data generated by a one-dimensional (1-D) model of ALD transport and reaction. We examined several ML algorithms including Bayesian optimization, physics-inspired optimization, and random optimization. We interfaced the ML code to our ALD system and allowed the algorithm to optimize the ALD process using in situ QCM measurements and observed rapid convergence, as predicted by our simulations. In short, we had designed the quartz crystal microbalance (QCM) sensor array hardware, designed and fabricated data collection electronics, created software to implement ML in our existing ALD reactor, developed a set of ML approaches for simultaneous development and comparison of different optimization algorithms, and successfully performed the first ML optimization of an ALD process.

\section{MATERIALS AND CHEMISTRY}

\begin{tabular}{|l|l|}
\hline LDRD \# 2019-0205 & Materials and Chemistry $\$ 1,551,718$ \\
\hline Project Title: & Ion Transport in Heterogeneous Materials \\
\hline Investigators: & $\begin{array}{l}\text { G. Brian Stephenson } \\
\text { Maria K. Chan, Hua Zhou, Michael J. Davis, Jeffrey A. Eastman, Peter Zapol, } \\
\text { Mark Antonio, Subramanian Sankaranarayanan }\end{array}$ \\
\hline
\end{tabular}

\begin{abstract}
Understanding how to control transport of ions in nanostructured liquids and solid electrolytes is fundamental to energy science and provides an opportunity to improve energy technologies, yet there are few direct studies of ion dynamics and its relation to heterogeneity. X-ray photon correlation spectroscopy (XPCS) at higher speeds and at interfaces is now becoming possible with improved detectors and coherent X-ray sources. We are extending the high-speed frontier of XPCS to the microsecond scale needed to observe dynamics in fluids and we are extending XPCS analysis to extract the higher-order correlations needed to characterize transition states. We used high-speed XPCS for extensive studies of several systems relevant to chemical separations, as well as for preliminary studies of electrolyte systems relevant to energy storage technology. In fact, we carried out the first XPCS
\end{abstract}


studies of ion dynamics in liquids and at solid interfaces, measuring correlation times below 100 microseconds. In addition, much progress has been made in developing coarse-grained molecular dynamics (MD) simulations, speckle analysis, free energy computations, and further advancing MD capabilities. The advances in simulations now allow detailed modeling of atomic-scale dynamics over the time and length scales critical to ion transport. We have also demonstrated the first simulations of ion dynamics, as well as exploring machine learning $(\mathrm{ML})$ approaches to optimize potentials and discover hidden correlations. What we learn will help predict and synthesize new heterostructures that enable improved control of ion flow along and through interfaces. Lastly, improvements made in computational infrastructure at the Advanced Photon Source (APS) for real-time analysis of Bragg XPCS data contributes to the efficacy of our overall approach.

\begin{tabular}{|l|l|}
\hline LDRD \# 2019-0433 & Materials and Chemistry $\$ 344,142$ \\
\hline Project Title: & Coupling Data Science and in situ Multi-Modal Probes for Materials Synthesis \\
\hline Investigators: & $\begin{array}{l}\text { Richard E. Wilson } \\
\text { Maria K. Chan, Uta Ruett, Logan Ward }\end{array}$ \\
\hline
\end{tabular}

\begin{abstract}
In contrast to the robust predictive frameworks that guide the synthesis of molecules, there are no such rubrics for the synthesis of materials. This is in part because the numbers of possible nucleation, growth, and restructuring steps involved in the synthesis of a material contribute to an exceptionally complex energy landscape. To navigate and map these complex landscapes, we attempted to couple emerging capabilities in data science with multi-modal in situ observations of synthesis reactions. We therefore designed a multi-modal reaction cell for use at the Advanced Photon Source (APS) that couples acquisition of X-ray scattering and Raman spectroscopy data. Initial Raman experiments used an off-line Raman microscope. Those data were analyzed to optimize collection strategies when at the APS. Upgrades to the Raman spectroscopic hardware at APS were made to enhance data quality at the beamline. We experimented with synthesis reactions that occur in molten alkali metal hydroxides, or fluxes, using a combination of in situ synchrotron-based X-ray probes and Raman spectroscopy. Data science techniques, such as unsupervised and supervised machine learning (ML), were planned to determine when reaction steps occur and to extract atomistic structures and determine reaction pathways from this deluge of multimodal data. As it happened during the active period of his project, COVID-19 restrictions affecting access to APS, as well as the complexity of the chosen materials system, slowed our progress. Nevertheless, applicability of data science in developing the science of materials synthesis was brought closer to realization.
\end{abstract}




\title{
PHYSICAL SCIENCES AND ENGINEERING
}

\begin{tabular}{|l|lc|}
\hline LDRD \# 2019-0386 & Physical Sciences and Engineering & \$716,403 \\
\hline Project Title: & Mineralogy Inspires Physics & \\
\hline Investigators: & Michael R. Norman \\
\hline
\end{tabular}

\begin{abstract}
Quantum materials have the potential to revolutionize technology. Materials exhibiting topologically significant properties that could be exploited for future technologies, such as topologically protected quantum computation, are of particular interest. Chemically doped variants that could exhibit hightemperature superconductivity are also of interest. Mineralogical databases can be used to identify candidates for new quantum materials that display either quantum spin liquid behavior or unconventional superconductivity. Electronic structure calculations have the potential to identify which of these materials have the greatest potential to exhibit novel quantum behaviors for future studies. We have performed such calculations in collaboration with colleagues at Northwestern University in order to identify which materials have the most potential to exhibit novel quantum behaviors for future studies. We found a potential pressure-induced quantum spin liquid phase in the mineral potassium iron jarosite as well as the possibility of a sawtooth spin chain in the mineral bobkingite. We encourage experimentalists to synthesize and study these materials.
\end{abstract}

\begin{tabular}{|l|l|}
\hline LDRD \# 2020-0164 & Physical Sciences and Engineering $\$ 268,729$ \\
\hline Project Title: & Accelerators Against Cancer \\
\hline Investigators: & $\begin{array}{l}\text { Jerry A. Nolen } \\
\text { Matthew R. Dietrich, Brahim Mustapha, Junqi Xie, David Rotsch, } \\
\end{array}$ \\
& Jeongseog Song, Peter Mueller, Matthew D. Gott, Robine De Kruijff \\
\hline
\end{tabular}

\begin{abstract}
Current cancer therapies place great strain on the human body resulting in severe side effects due to their non-specific targeting of healthy and cancer cells alike. Enhancing chemotherapy with radiation therapy reduces the overall drug doses needed, which could help reduce patient distress. Our thrust is to demonstrate applications of medical isotopes, such as in radio-assisted chemotherapy. Cisplatin is a platinum-centered molecule that directly interacts with deoxyribonucleic acid (DNA). Auger-electron emitters release a potent radiation dose over a range less than a cell's width, minimizing damage to surrounding cells. Auger-emitting platinum isotopes were made by both direct and indirect reaction pathways. Separation studies were performed to isolate platinum from osmium target material through mild heating, enabled by the unique properties of osmium tetroxide. We have made considerable progress towards measuring the first low-energy Auger spectrum of medically relevant isotopes with an Auger radioisotope microscope apparatus. A second isotope of interest is Astatine-211. It is a promising alpha particle emitter suitable for targeted alpha therapy. Unfortunately, the impact of Astatine-211 has been constrained by its limited availability. An attractive approach to overcoming that issue is the
\end{abstract}


Radon-211/Astatine-211 generator. The generator allows Astatine-211 to grow and reach maximum radioactivity at 14.5 hours. Moreover, over $80 \%$ of the maximum activity level is still available on the generator after 24 hours. This permits delivery of a generator to remote locations, significantly extending time for distribution. Using Radon-222 as a surrogate, we assembled an apparatus to investigate the transfer and trapping of Radon-222.

\title{
QUANTUM INFORMATION SCIENCES
}

\begin{tabular}{|l|l|}
\hline LDRD \# 2019-0358 & Quantum Information Sciences \\
\hline Project Title: & A Scalable Toolkit for Noisy-Qubit Simulation \\
\hline Investigators: & $\begin{array}{l}\text { Hal J. Finkel } \\
\text { Martin Suchara, Matthew Otten }\end{array}$ \\
\hline
\end{tabular}

\begin{abstract}
Quantum computing, which promises to revolutionize the computing landscape, is a near-term reality: quantum computers with nearly 50 qubits are expected to appear this year and 100-qubit machines are possible within the next several years. The goal of this project, at a high level, was to take the Argonnedeveloped QuaC (open-Quantum-systems in C) simulation software and create a toolkit usable for investigating different quantum information science (QIS) technologies. In particular, a toolkit for noisy qubit simulation that will enable co-design between quantum algorithms and materials research, including the customization of partial error correction to realistic qubit properties, using both timedependent and time-independent operators. To that end, we wrote a Python interface for QuaC and created a new web site. We developed a plugin to IBM's QisKit quantum computing framework allowing the comparison of circuits on IBM's hardware to the QuaC simulation results. We validated the plugin interface and the noise models against IBM's simulator. Due in part to this effort, QuaC has been selected by the DOE/ASCR-funded AIDE-QC (Advancing Integrated Development Environments for Quantum Computing) project for integration into the XACC (eXtreme-scale ACCelerator) quantumsystem-simulation framework.
\end{abstract}

\begin{tabular}{|l|l|}
\hline LDRD \# 2019-0360 & Quantum Information Sciences $\$ 506,322$ \\
\hline Project Title: & Quantum State Engineering in Strong-Coupling Circuits using Floquet Theory \\
\hline Investigators: & $\begin{array}{l}\text { Ivar Martin } \\
\text { Aashish Clerk, Salman Habib }\end{array}$ \\
\hline
\end{tabular}

\footnotetext{
Abstract

Quantum information processing has enjoyed explosive growth over the past few years as the next computing paradigm of great importance to national security. Superconducting technology has emerged as a leading technology in this arena. Next-generation quantum information platforms place a high demand on quantum coherence as a resource, and necessarily operate far away from statistical
} 
equilibrium. Our goal is to develop theoretical methods for the efficient description of driven superconducting quantum circuits in the regime of ultra-strong light-matter interactions and/or strong driving. We investigated if new, dynamical quantum states, suitable as potential quantum information carriers, can be stabilized away from equilibrium, under periodic drive. We developed an effective stroboscopic Hamiltonian description of the driven continuum cavity problem. We have also focused on periodically driven multimode cavities. We have developed a quantum description of focusing phenomena in a single multimode and in connected multiple single mode cavities. We successfully identified a series of such states that can be engineered in generic one-dimensional coupled systems such as a chain of connected superconducting cavities. We found that when such systems are driven in a spatially inhomogeneous manner---notably including drive on their boundaries only---a series of quantum wave packets emerge, which traverse the chain back and forth, yet never disperse. In other words, they maintain their spatial sharpness indefinitely. Such non-dispersing wave packets are remarkable and cannot exist in undriven systems. Their spatial sharpness makes them ideal entities for information injection and readout, and their ability to traverse the entire system back and forth while maintaining coherence opens up the possibility for them to serve as a "bus" of sorts, over which quantum information can be shuttled across an entire system.

\begin{tabular}{|l|l|}
\hline LDRD \# 2019-0377 & Quantum Information Sciences $\$ 480,687$ \\
\hline Project Title: & Long-Lived Erbium Qubits in Silicon-Based Semiconductor Materials \\
\hline Investigators: & $\begin{array}{l}\text { Tijana Rajh } \\
\text { Tian Zhong, David Awschalom }\end{array}$ \\
\hline
\end{tabular}

\begin{abstract}
Scalable technology for long-lived qubits underpins quantum engineering. Erbium rare-earth ions constitute a unique system that simultaneously possesses second-long spin coherence and fibercompatible telecom optical emissions. We proposed a new rare-earth qubit technology in epitaxial oxides on silicon and silicon carbide obtained by molecular beam epitaxial growth. We assembled the tools for spectroscopic characterization of samples at millikelvin temperatures. We doped the isotope erbium-167 $\left({ }^{167} \mathrm{Er}\right)$ into bulk polycrystalline and epitaxial films of yttrium oxide $\left(\mathrm{Y}_{2} \mathrm{O}_{3}\right)$ with the desired site occupancy, as well as electron paramagnetic and spin resonance behaviors, and optical emission properties. This structure is where we seek to engineer long-lived coherent erbium-167 hyperfine qubits. We observed that the influence of the film-substrate interface and of lattice (mis)matching of $\mathrm{Er}$ to the yttrium oxide perturb the local Er environment. We extended our study to include lanthanum yttrium oxide as erbium's host lattice and fabricated silicon (Si) waveguides or resonators for placement on the erbium-doped films to enhance optical absorption and improve photo emission rate. Our demonstration of long coherence times of optically addressable ${ }^{167} \mathrm{Er}$ qubits has been our most promising achievement to date. Such simultaneous long coherences in both optical and spin degrees of freedom are not found in other rare-earth doped oxide crystals. The erbium-on-silicon architecture should enable scalable platforms for quantum information processing and storage.
\end{abstract}




\begin{tabular}{|l|l|}
\hline LDRD \# 2019-0381 & Quantum Information Sciences $\$ 493,815$ \\
\hline Project Title: & $\begin{array}{l}\text { A Novel Readout for Large Arrays of Superconducting Nanowire Single Photon } \\
\text { Detectors }\end{array}$ \\
\hline Investigators: & $\begin{array}{l}\text { Clarence L. Chang } \\
\text { Thomas W. Cecil, Valentine Novosad, Gensheng Wang }\end{array}$ \\
\hline
\end{tabular}

\section{Abstract}

Superconducting nanowire single photon detectors (SNSPDs) are a core technology for quantum information science. We sought to provide a new capability, enabling these detectors to be implemented in large-scale applications by developing a new approach to reading out SNSPDs. The proposed concept uses superconducting resonators that are intrinsically multiplexable to large channel counts and are a favored detector technology for quantum information sciences (QIS). The resonatorbased readout would use materials with large non-linear kinetic inductance to transduce the SNSPD signal into shifts in resonator frequency. We have designed and simulated candidate sensor layouts; fabricated mounting hardware for device operation; and fabricated and characterized initial devices. We measured a set of resonant structures, characterizing the dependence of resonator frequency and quality factor on coupled thermal energy by varying the device temperature. We have studied a number of readout concepts and carried out the first stages of materials synthesis, resonator design and fabrication, and device testing. The resonators work well and provide data to parameterize relevant properties for device modeling. Conceptual studies of devices using novel materials (such as titanium nitride) were carried out. Noise and timing performance for various coupling architectures were also calculated.

\begin{tabular}{|l|l|}
\hline LDRD \# 2019-0466 & Quantum Information Sciences $\$ 132,208$ \\
\hline Project Title: & $\begin{array}{l}\text { A User-Friendly AI Software Toolkit for Molecular Modeling and Design of } \\
\text { Quantum Materials }\end{array}$ \\
\hline Investigators: & Subramanian Sankaranarayanan \\
\hline
\end{tabular}

\section{Abstract}

The artificial intelligence (Al) tool will advance synthesis science and accelerate the discovery and design of correlated material phases with exotic properties. This project involves use of machine learning (ML) toolkits for multi-fidelity scale bridging and also involves theoretical development of a framework for quantum information science (QIS). We had specific interest in the area of dissipative quantum systems as well as in the role of noise in quantum systems. We conducted a survey of deep learning (DL) methods for material science with extra small datasets. We noted that there is a lack of literature exploring behavior of neural networks (NNs) in a small dataset setting. We worked on developing such techniques. To fine-tune NNs for such applications, we identified and developed a theoretical background for specific techniques for all aspects of DL: data preparation, network initialization, gradient descent methods, and network regularization techniques. To provide experimental evidence for the developed theoretical guidelines, we worked on predicting properties of a specific class of two- 
dimensional (2D) topological materials, using density functional theory (DFT) calculations for such materials as training data. We created DL models that would outperform in accuracy any available classical ML algorithms, which is a groundbreaking result, considering the size of the dataset. Apart from outperforming classical ML algorithms in terms of accuracy, we created a NN that would allow prediction of multiple material properties at once - something that is difficult or even impossible to do using ML algorithms typically used in material science. Moreover, our architecture works well even when training data has varying accuracy from one material property to another. Finally, adding to our plan, we also explored the development of quantum computing algorithms in application to ML. In particular, we explored the applicability of hypothetical general quantum computers for DL.

\title{
SECURING ENERGY AND CRITICAL RESOURCES
}

\begin{tabular}{|l|l|}
\hline LDRD \# 2018-089 & Securing Energy and Critical Resources $\quad \$ 738,222$ \\
\hline Project Title: & $\begin{array}{l}\text { Optimizing Urban Transportation Systems Energy Using Large-Scale Simulation } \\
\text { and Machine Learning }\end{array}$ \\
\hline Investigators: & $\begin{array}{l}\text { Aymeric Rousseau } \\
\text { Zhi Zhou, Joshua A. Auld }\end{array}$ \\
\hline
\end{tabular}

\begin{abstract}
Our project develops a tool to allow the automotive and electric power industry to locate, size, and design control for a plug-in electric vehicle (PEV) fast charging infrastructure that maintains both economic viability and grid reliability while leveraging connected EV storage to increase system resiliency. We completed the first stage of our project by expanding the core capability of the transportation system simulation tool, POLARIS, and the Argonne Least-Cost Energy Framework (ALEAF), a power system simulation framework. With the coupled simulation tools, we completed the development of an open-loop linkage between POLARIS and ALEAF. The initial results have demonstrated the capability and potential application of the modeling framework to investigating the impact of the high penetration of PEVs on short-term and long-term power system operation and planning. We have added new capabilities to POLARIS and ALEAF while strengthening the workflow. Key emphasis included integration of traveler charging behavior (i.e., when to charge) along with implementation of a machine learning $(\mathrm{ML})$ model to estimate vehicle energy consumption in POLARIS and to model distribution networks in ALEAF. Particularly, the ALEAF has been extended with a capability to model charging infrastructure planning decisions with consideration of distribution network constraints. Based on the coupled POLARIS and ALEAF, we have completed analysis and model extension on both the transmission and distribution networks. For the transmission network, we completed analysis of the impact of future adoption scenarios on power grid expansion and planning with a planning horizon of 15 years under various renewable energy policies. For the distribution network, we extended the capability to model the impact of traveling and charging demand on charging infrastructure planning with consideration of distribution network operational constraints and power quality.
\end{abstract}




\begin{tabular}{|l|l|}
\hline LDRD \# 2018-097 & Securing Energy and Critical Resources $\quad \$ 516,702$ \\
\hline Project Title: & Mission-Driven Unmanned Aerial System (UAS) Design Center \\
\hline Investigators: & $\begin{array}{l}\text { Thomas Wallner } \\
\text { Sibendu Som, Joseph J. Adduci, Dominik A. Karbowski, Yuki Hamada }\end{array}$ \\
\hline
\end{tabular}

\begin{abstract}
This project focuses on understanding and predicting mission-specific power and energy requirements and developing mission-driven unmanned aerial system (UAS) designs. The main technical goal was to develop modeling capabilities for evaluating and predicting mission-specific power and energy requirements and ultimately developing mission-driven UAS designs. These are key enabling steps towards assessing the potential and implications that large-scale deployment of UASs could have on securing energy and critical resources in the future. Interconnected technical challenges include energy consumption, noise, flight time and payload. We addressed developing a tool to evaluate potential UAS design options including battery powered as well as hybrid architectures based on mission driven characteristics and optimizing the trade-offs through targeted aerodynamics design verification. Autonomie is Argonne's system simulation tool for ground vehicle energy consumption and performance analysis. We worked to replicate the success of Autonomie in the area of aircraft, and UAVs in particular, with "Aeronomie." Aeronomie has the ability to run analyses where power plant type and size, high-level aerodynamic characteristics, payload, mission profiles, and many other variables are parameters. It will give researchers the opportunity to explore new system designs and new missions. The efficiency of a fixed-wing or a single/multi-rotor UAS and the noise from its propellers can be improved by perfecting the aerodynamics of its wings or blades. Noise-generating structures (such as vortices and separations) are extremely challenging to simulate and analyze. However, we pursued use of high-fidelity computational fluid dynamics (CFD) techniques and high-performance computing (HPC) tools to make tractable analytical designing of UASs.
\end{abstract}

\title{
THE UNIVERSE AS OUR LAB (ULAB)
}

\begin{tabular}{|l|l|}
\hline LDRD \# 2018-053 & Universe as Our Lab (ULab) \\
\hline Project Title: & $\begin{array}{l}\text { A High-Pressure Xenon Gas Time Projection Chamber for Next Generation } \\
\text { Neutrinoless Double Beta Decay Search Experiments }\end{array}$ \\
\hline Investigators: & $\begin{array}{l}\text { Corey J. Adams } \\
\text { Seamus P Riordan, Kevin G. Bailey }\end{array}$ \\
\hline
\end{tabular}

\begin{abstract}
Neutrinoless double beta decay (NLDBD) offers a window to new physics. It provides a sensitive probe into the mysteries of the neutrino with implications for unsolved questions in physics such as the observed excess of matter in the universe. High-pressure gaseous xenon (HPGXe) time projection chambers (TPCS) are a technology suited for searching for NLDBD, as they provide excellent energy resolution while simultaneously capturing event topology useful for background rejection. Our project explores the detection of ions rather than electrons in a xenon-136 HPGXe TPC to either discover NLDBD
\end{abstract}


or to set the best upper limit for this process. The Xe-136 isotope undergoes double beta decay with an exceeding long half-life ( $>10^{21}$ years). Drifted ions exhibit significantly less diffusion than electrons and offer improved spatial reconstruction of events. A TPC is an attractive component of any ton-scale gaseous xenon NLDBD detector, since the relevant signal is distinguished topologically from the single beta decays and gamma events. We have completed the mechanical design of the electrostatic field cage and have constructed a high voltage feedthrough. The electrostatic analysis of the field cage for a complete design precedes moving to its construction. We acquired a new pressure vessel for our tests of field cage components in high pressure gaseous xenon, plus new microchannel plate photomultipliers for light detection. Our work leads to supplying a critical component to the Neutrino Experiment with a Xenon Time Projection Chamber (NEXT) collaboration.

\begin{tabular}{|l|l|}
\hline LDRD \# 2019-0034 & Universe as Our Lab (ULab) $\$ 156,884$ \\
\hline Project Title: & $\begin{array}{l}\text { Cooling the Cooler: Using Optical Stochastic Cooling to Prepare Cold Electrons } \\
\text { for Cooling Hadrons in the Electron-Ion Collider }\end{array}$ \\
\hline Investigators: & Alexander A. Zholents \\
\hline
\end{tabular}

\begin{abstract}
"Cooling" of hadron beams in the electron ion collider (EIC), to be built at Brookhaven National Laboratory, will allow a multifold increase of the integral luminosity. Therefore, development of a cooling technique applicable to the EIC is being pursued by several laboratories involved with EIC design. The task is challenging because known cooling techniques require an intense electron beam with stateof-the-art low emittance and energy spread. The electrons interact with hadrons in the process of cooling by absorbing their excess energy. This leads to an increase in the emittance and the energy spread of the electron beam. The main approach in the design of the electron source for hadron cooling is to use a state-of-the-art recirculating linac and to discard the electrons after just one interaction. Here, we take a radically different approach whereby we consider "cooling" the electrons back to their original state and re-using them. To do that, we store the electrons in the storage ring, recycling them quasi-indefinitely, while maintaining the low emittance and low energy spread, using an optical stochastic cooling (OSC) method. We expanded the OSC method for cooling electron and positron beams. We have shown that a drastic increase in the bandwidth of a cooling system to 10 petahertz ( $\mathrm{PHz})\left(1 \mathrm{PHz}=10^{15} \mathrm{~Hz}\right)$ can be obtained by using an extreme ultraviolet (EUV) light, eliminating an optical amplifier and replacing refraction lenses with off-axis parabolic mirrors. This in turn paves the way for a few beneficial modifications of a stochastic cooling technique such as a deferred correction action and cascade amplified stochastic cooling. That results in an order of a magnitude shorter damping time than synchrotron radiation damping time. Nevertheless, this was not sufficient to maintain the low emittance and low energy spread intense electron beam that the EIC needs because of very strong intra-beam scattering.
\end{abstract}




\begin{tabular}{|l|lc|}
\hline LDRD \# 2019-0050 & Universe as Our Lab (ULab) \\
\hline Project Title: & Horn-Coupled Detectors for Future CMB Experiments \\
\hline Investigators: & $\begin{array}{l}\text { Thomas W. Cecil } \\
\text { Stephen Padin }\end{array}$ \\
\hline
\end{tabular}

\begin{abstract}
We aimed to advance the science and technology of superconducting microwave detectors by developing new techniques for coupling signals to an array of detectors using horns. Our immediate goal was to demonstrate performance suitable for the upcoming Cosmic Microwave Background Stage 4 experiment (CMB-S4). Horns will be used for most, if not all, CMB-S4 observing bands, because they can couple signals with very small beam and polarization errors, but existing horn coupling schemes allow too much signal to leak at the horn-to-detector-wafer interface; new ideas are needed to address the problem. The success of this work will result in a focal plane design with optimal pixel spacing for two of the CMB-S4 frequency bands. We developed designs for single-layer lumped-element kinetic inductance detectors sensitive to photons in the 220- and 270-gigahertz frequency bands by using electromagnetic software packages. Single-pixel devices were fabricated at Argonne's Center for Nanoscale Materials and characterized in an adiabatic demagnetization-refrigerator cryostat. Optical testing was performed to confirm optical coupling to the inductor antenna. We have identified and simulated a horn coupling scheme to reduce signal leakage at the horn to detector wafer interface and designed and tested orthomode transducers (OMT). We have fabricated prototype detector wafers modified for the coupling scheme. Our design of the wafer holder incorporating the new horn coupling scheme along with fabrication and testing of a horn and wafer assembly has been carried out.
\end{abstract}

\title{
DIRECTOR'S COLLABORATIONS
}

\begin{tabular}{|l|lc|}
\hline LDRD \# 2018-110 & Director's Collaborations & $\$ 37,326$ \\
\hline Project Title: & Negative Capacitance in Ferroelectric Nanostructures \\
\hline Investigators: & Saidur Rahman Bakaul \\
\hline
\end{tabular}

\begin{abstract}
This project studied local (nanometer spatial resolution) ferroelectric properties, particularly capacitance and charge transport, in complex oxide ferroelectric materials. Electrostatic force microscopy was used to probe the samples. Our approach was to utilize epitaxial thin films at the nanoscale where in single-domain samples, sharp switching of polarization states not complicated by domain wall motion, is expected. We found that the observed capacitance was quite strongly dependent on the distance separating the probe tip and the sample surface. Although this strong effect precluded aspects of the desired measurements, we were successful with regard to development of the technique and its instrumentation in that our data could be understood and analyzed using a tip-sample electrostatic interaction model.
\end{abstract}




\begin{tabular}{|l|lc|}
\hline LDRD \# 2020-0172 & Director's Collaborations & $\$ 27,730$ \\
\hline Project Title: & Computationally Evolved 2D Peptide Assemblies & \\
\hline Investigators: & $\begin{array}{l}\text { Harry C. Fry } \\
\text { Subramanian Sankaranarayanan }\end{array}$ \\
\hline
\end{tabular}

\begin{abstract}
The goal of this project was to identify parameters for optimizing biomolecular materials design via an experimental feedback loop. Data fed back from experiment train artificial intelligence (AI) tools for further iterations. In principle, the strategy can be extended to any synthetic materials process that can be automated. In addition, two-dimensional peptide materials facilitate the specific placement of porphyrin molecules in an ordered array. Peptide assembly can bind and organize heme molecule which is responsible for multiple functions (oxygen-carrying, catalysis, electron transfer) in many living organisms. We intended to find a computational method that would lead to the discovery of short peptide sequences that would self-assemble into 2D sheets for interfacing with other materials. We succeeded in using molecular modeling for peptide materials discovery. We explored the coarse-grained molecular modeling of a series of peptide amphiphiles where the length of the terminal alkyl chain was varied from $8,12,16$, and 18 carbons. By varying the alkyl chain, we discovered something new. The self-assembly mechanism of the series of peptides was dependent on the alky chain length, such that the $\mathrm{pH}$ required to trigger the assembly was well tuned. This was validated via coarse-grained molecular modeling. Through comparison with experiment, we discovered the assembly limitation of the peptides was controlled by not only the length of the alkyl chain, but also the charge of the amino acid adjacent to the hydrophobic portion of the amino acid. This finding is critical for efforts in discovering new peptides for binding light activated or redox active chromophores like metalloporphyrins. By employing artificial intelligence and machine learning, we also explored the generation of sequence libraries of short peptides that self-assemble into any structure. We developed an $\mathrm{Al} /$ machine learning (ML) algorithm that utilizes a Monte Carlo Tree Search Algorithm in conjunction with molecular dynamics (MD) simulations to determine the probability of whether a pentapeptide sequence will assemble into some structure or remain soluble. Al can determine which peptides are worth exploring for selfassembly by scoring the peptide with the resulting aggregation propensity from MD simulations and balancing that with a hydrophobicity score. Essentially, a peptide with a high aggregation propensity that is hydrophilic will score high. We synthesized a selection of peptides and found that the Al-expert designed peptides yielded more diverse sequences (i.e., employed more amino acids) than those proposed by human experts whose suggestions were solicited for comparison.
\end{abstract}




\title{
NAMED FELLOWS
}

\begin{tabular}{|l|l|}
\hline LDRD \# 2017-093 & Named Fellows \\
\hline Project Title: & $\begin{array}{l}\text { Beehive: A Dynamic Execution Environment for Performance, Power, and } \\
\text { Resilience on Extreme-Scale Computing Systems }\end{array}$ \\
\hline Investigators: & $\begin{array}{l}\text { Min Si } \\
\text { Pavan Balaji }\end{array}$ \\
\hline
\end{tabular}

\section{Abstract}

High-performance computing (HPC) is playing an important and ever-increasing role in many scientific domains. The increasing complexity of HPC hardware architectures (i.e., processor and memory) aggravates irregularities in data communication across parallel processors. The resulting imbalanced computational load leads to extreme resource overhead during simulation of scientific applications. Beehive's dynamic execution model can balance performance, resilience, and power awareness for irregular applications on advanced HPC platforms. The core concept of Beehive is based on overdecomposition. Within Beehive, the decomposition of the computational work is not bound by the available hardware processing units. Each physical processing unit can have hundreds or thousands of work units assigned to it, and the Beehive runtime will dynamically manage these work units to optimize for performance, fault resilience, and power. It utilizes a combination of operating system and runtime techniques in order to dynamically balance workloads and hide communication overhead. Beehive provides transparent performance optimizations to message passing interface (MPI) implementations on upcoming supercomputers such as the Argonne Aurora supercomputer. It has enabled efficient quantum chemistry simulation through dynamic communication progress and inter-process workload balancing and accelerated particle transport simulation through flexible address-space sharing. We expect Beehive will be adopted by the systems of the major HPC vendors.

\begin{tabular}{|l|l|}
\hline LDRD \# 2017-157 & Named Fellows \\
\hline Project Title: & $\begin{array}{l}\text { Transport and Photophysical Processes in Hybrid Perovskites for Energy } \\
\text { Applications }\end{array}$ \\
\hline Investigators: & $\begin{array}{l}\text { Peijun Guo } \\
\text { Richard D. Schaller }\end{array}$ \\
\hline
\end{tabular}

\begin{abstract}
Hybrid perovskites are an emerging class of semiconductors that are solution processable, earthabundant, and exhibit excellent performance in light-harvesting and light-emitting applications and provide complementary functionalities to the traditional oxide perovskite materials. A better understanding of the structure-property relationships within the hybrid perovskites will benefit the photovoltaic and optoelectronic communities by suggesting ways to optimize the composition and processing of materials, as well as the design of devices. As hybrid perovskites exhibit a diverse range of physical properties, chemical variabilities and structural diversities, we expect that the results of our research will help facilitate their application in ferroelectricity and magnetism. Our project's goal was to
\end{abstract}


understand the optical physics and the electron and lattice dynamics of hybrid perovskites. To accomplish that, we pioneered a new spectroscopic approach for this study based on ultrafast infrared vibrational excitation of the lattice. Using this technique, we revealed critical information related to how energy moves between the two sub-lattices in methylammonium lead iodide $\left(\mathrm{CH}_{3} \mathrm{NH}_{3} \mathrm{Pbl}_{3}\right)$, a prototypical hybrid perovskite material. Via the transient absorption measurements, employing an infrared pump pulse tuned to a methylammonium vibrational resonance, we observed slow, nanosecond-long thermal dissipation from the selectively excited organic mode to the inorganic sublattice. The resulting transient electronic signatures, during the period of thermal-nonequilibrium when the induced thermal motions are mostly concentrated on the organic sublattice, reveal that the induced atomic motions of the organic cations do not alter the absorption or the photoluminescence response of the material beyond thermal effects. Our results therefore suggest that the attractive optoelectronic properties mainly derive from the inorganic lead-halide framework.

\begin{tabular}{|l|l|}
\hline LDRD \# 2017-158 & Named Fellows \\
\hline Project Title: & Real-time Control of Urban Drainage Systems \\
\hline Investigators: & $\begin{array}{l}\text { Sara Rimer } \\
\text { Charles M. Macal }\end{array}$ \\
\hline
\end{tabular}

\begin{abstract}
Our goal has been to improve the understanding and employment of cyber-physical systems for urban infrastructure, specifically the development of "smart" urban watersheds capable of adaptively responding to dynamic and uncertain weather events. We seek to advance technologies, capabilities, and insights into the real-time sensing and control of the watersheds via remotely deployed sensors, improved stormwater modeling techniques, and utilization of novel data methods. We led development of a suite of benchmark stormwater networks for testing real-time control algorithms for watershedlevel control, including open-source computational code hosted online in order to encourage collaboration across an open-source research consortium. We built real-time water quality sensors for deployment in the Chicago Area Waterway System (CAWS). They will be used in conjunction with autosamplers for collecting real-time data on the fecal indicator bacteria dynamics of the waterway. This research will augment the Argonne Microbiome Study by providing bacteriological analyses at various microbiome measurement locations in the CAWS at refined time scales during a storm event, thereby enhancing our understanding of the microbial dynamics of overall microbial health. We also began development of a computational predictive model of the CAWS using historical water quality data. The built sensors will be deployed for initial collection of real-time water quality data, and a predictive model will continue to be in development in conjunction with the data collected via the sensor systems.
\end{abstract}




\begin{tabular}{|l|l|}
\hline LDRD \# 2018-051 & Named Fellows $\$ 622,945$ \\
\hline Project Title: & $\begin{array}{l}\text { High-Efficiency, Low-cost ORR \& OER Catalysts Derived from Metal-Organic } \\
\text { Frameworks }\end{array}$ \\
\hline Investigators: & Lina Chong \\
\hline
\end{tabular}

\begin{abstract}
A low-temperature reversible fuel cell (RFC) will play a key role in future energy storage for distributed power. Unlike a battery, an RFC stores excess grid electric energy in the form of hydrogen and oxygen through electrochemical water splitting and, when needed, recombines them electrocatalytically to produce electricity. An RFC consists of two components - a proton-exchange membrane fuel cell (PEMFC) and a proton-exchange membrane water electrolysis (PEMWE) system. Both the PEMFC and the PEMWE systems contain two electrodes separated by a proton-exchange membrane that transports $\mathrm{H}+$ ions between the electrodes during the electrocatalytic reactions. The acidic environment and high polarization voltage, however, challenges the electrode catalyst activity and durability. This is particularly the case for the RFC electrode at which the oxygen evolution reaction (OER) takes place during water splitting and where the oxygen reduction reaction (ORR) occurs during electricity production. Catalysts are needed to promote the efficiency of OERs and ORRs. Currently, platinum group metals (PGM) such as platinum (Pt) and iridium (Ir) are the catalyst materials of choice. Their high cost and limited reserves, however, are a barrier to widespread implementation of RFCs. In this project, we have developed a new class of high-efficiency and low-cost ORR/OER catalysts for PEMFC/PEMWE using a transition-metal-based metal-organic framework (MOF) as the precursor. We demonstrated a new ORR catalyst based on the synergistic interaction between ultralow loading platinum supported over a MOF derived precious metal free catalytic substrate. Similarly, we have made some significant progress in OER catalyst development and demonstrated a highly efficient precious metal free catalyst operable in the acidic environment. Further development work resulted in highly efficient, low-cost ORR and OER catalysts. Their activities and durability under practical operating conditions have been tested. We also performed systematic studies on the synergistic ORR/OER mechanisms through in situ X-ray absorption spectroscopy experiments at Argonne's Advanced Photon Source (APS). Substantially lowering the cost of these catalysts facilitates commercialization of RFCs and offers advantages to their manufacturers and to their end users.
\end{abstract}

\begin{tabular}{|l|lc|}
\hline LDRD \# 2018-052 & Named Fellows & \$373,788 \\
\hline Project Title: & Exploring Lossy Quantum Computation & \\
\hline Investigators: & $\begin{array}{l}\text { Matthew Otten } \\
\text { Mi Sun Min }\end{array}$ \\
\hline
\end{tabular}

\title{
Abstract
}

As a quantum computer carries out a computation, dephasing, decoherence, and decay (from interactions with the environment) will gradually cause noise and errors to accumulate. We pursued ways to deal with and simulate the errors that can occur in quantum computation and other problems of relevance to quantum information science (QIS). How these errors affect quantum algorithms was 
studied. Understanding the limits of a specific algorithm or a specific quantum computing platform requires detailed, careful simulations. Our focus was on the study of lossy (noisy) quantum computation from several angles, including novel algorithm development for noisy quantum computers and the study of the physical systems that makeup noisy quantum computers. We continued to lead development of the QuaC software, a parallel time-dependent quantum systems solver for simulating noisy quantum computers. QuaC has the flexibility to model quantum architectures at gate or pulse levels, allowing for studies of quantum networks and quantum sensors. To simulate open quantum systems, the Lindblad master equation was used. The equation is able to accurately describe a variety of quantum systems in the presence of noise. Our study dealt with a wide variety of aspects of the problem including: a novel quantum dynamics algorithm; a novel quantum machine learning algorithm; an investigation of trions and biexcitons in CdSe nanoplatelets; longrange energy propagation in coupled plasmon-quantum dot systems; helium electron bubble dynamics; an algorithm for calculating high-order exciton properties; coherent photon coincidence spectroscopy; various physical quantum memories; the use of stochastic optimizers for variational quantum algorithms; implementations of molecular dynamics on quantum computers; and techniques for benchmarking quantum computers using random unitary matrices.

\title{
INNOVATE
}

\begin{tabular}{|l|l|}
\hline LDRD \# 2019-0023 & Innovate \\
\hline Project Title: & Novel Production of Theranostic Precious Metal Radionuclides at ATLAS \\
\hline Investigators: & $\begin{array}{l}\text { Matthew Gott } \\
\text { David Rotsch, Jeongseog Song, Jerry Nolen }\end{array}$ \\
\hline
\end{tabular}

\begin{abstract}
Platinum-based chemotherapeutic agents are leading drugs for cancer therapy. Recently, iridium-based anti-cancer drugs have also been developed. Incorporation of radioactive isotopes of platinum or iridium into these agents has value for diagnostic gamma-ray imaging to quantify uptake, retention, and clearance of these drugs. Furthermore, when incorporated at radiotherapeutic levels, they have the potential for effectively killing cancer cells. This approach depends on high specific activity and high radionuclidic purity, criteria that have been difficult to achieve for these isotopes. Producing isotopes using rare beams only available at the Argonne Tandem Linac Accelerator (ATLAS) is scientifically innovative and is an emerging technology for isotope production. We fabricated target stacks consisting of four to eight foils of thin rhenium or tungsten interlaced with bismuth monitoring foils for irradiation to measure production cross-sections as a function of energy. We performed several irradiations at ATLAS of the natural abundance target metals and determined cross-sections for the platinum isotope through the ${ }^{\text {nat }} \mathrm{Re}\left({ }^{6} \mathrm{Li}, \mathrm{xn}\right)^{188} \mathrm{Pt}$ nuclear reaction and for the iridium isotope through the ${ }^{\mathrm{nat}} \mathrm{W}\left({ }^{6} \mathrm{Li}, \mathrm{xn}\right){ }^{186} \mathrm{Ir}$ reaction. The typical irradiation lasted six hours and utilized a $70 \mathrm{MeV}^{6} \mathrm{Li}$ beam of 20 particle nanoAmpere intensity. Cross-section data for lithium bombardment are rare, and none were known for these production pathways prior to our work. Our data revealed the optimal energy to best produce the desired products. These data will be useful for optimization of isotope production rates. The demonstrated unique reaction pathways show promise for producing a pure product that is not available through other production routes.
\end{abstract}




\begin{tabular}{|l|l|}
\hline LDRD \# 2019-0250 & Innovate \\
\hline Project Title: & $\begin{array}{l}\text { The Development of a High-throughput Mass Spec-based Method for } \\
\text { Biocatalyst Engineering }\end{array}$ \\
\hline Investigators: & $\begin{array}{l}\text { Gyorgy Babnigg } \\
\text { Anindita Basu, Marie-Francoise Gros }\end{array}$ \\
\hline
\end{tabular}

\section{Abstract}

Biocatalysts are used in a number of industries. They can replace traditional homogenous chemical catalysts, providing a higher turnover rate and superior selectivity under environmentally friendly conditions. Biocatalysts are increasingly replacing many traditional catalysts, especially those used in the pharmaceutical industry. They are optimized by protein engineering. While classical methods can survey only a limited set of variants, our project aimed to develop a high-throughput method that surveys a much larger repertoire of enzyme modifications. Novel enzymes may have applications for biomanufacturing, biofuels and biosynthesis of high added-value molecules. We developed a microfluidic chip capable of splitting aqueous droplets into daughter droplets and channeling them into two separate flows, one for mass spectrometry and the other for sorting. The mass spectrometer monitors enzyme reactions. We have cloned, expressed and purified 96 enzyme variants from two families. One family of enzymes is important in bioproduction, and the other in the degradation of lignocellulosic biomass. About $30 \%$ of the proteins appeared to be soluble and directly useable when expressed in E. coli. Our mid-throughput method was further optimized and used to measure bioproduct titers for more than 5,000 samples to date. This technique is now an integral part of the Agile Biofoundry at ANL.

\begin{tabular}{|l|l|}
\hline LDRD \# 2019-0328 & Innovate \\
\hline Project Title: & Active Plasmonics using Epitaxial Thin Films of Dirac Semimetals \\
\hline Investigators: & $\begin{array}{l}\text { Anand Bhattacharya } \\
\text { Richard Schaller }\end{array}$ \\
\hline
\end{tabular}

\footnotetext{
Abstract

This project sought to break new ground in active terahertz $(\mathrm{THz})$ plasmonics by creating novel plasmonic structures using a new class of materials, topological semimetals. For example, one sample type investigated was the theoretically predicted three-dimensional non-magnetic bismuth-antimony Weyl semimetal $\mathrm{Bi}_{1-\mathrm{x}} \mathrm{Sb}_{\mathrm{x}}$. Another structure that was considered was epitaxial thin films of threedimensional graphene. The unique response of such materials to external fields, low carrier densities, and high mobilities should make them frequency-agile and field-tunable. We have created large-area epitaxial films and patterned structures such that the properties of the plasmons (and associated polaritons) can be controlled by using their lateral structure. We also created interfaces of these materials with magnetic thin films to understand the interaction of spin currents with the optical modes in the $\mathrm{THz}$ regime. This research will be of potential benefit to terahertz spectroscopy, imaging, sensing, and $\mathrm{THz}$ sources/detectors.
} 


\begin{tabular}{|l|l|}
\hline LDRD \# 2020-0174 & Innovate \\
\hline Project Title: & $\begin{array}{l}\text { Machine Learning Methods for Spectral Data from X-ray Transition Edge Sensor } \\
\text { Arrays }\end{array}$ \\
\hline Investigators: & Tejas Guruswamy \\
\hline
\end{tabular}

\title{
Abstract
}

Arrays of superconducting transition edge sensor (TES) calorimeters and other similar low-temperature detectors provide high-resolution X-ray spectroscopic data, surpassing semiconductor-based detectors. However, significant manual parameter tuning is required to achieve the best energy resolutions. Improving reproducibility and reducing the manual effort needed to analyze the data would substantially improve their utility. To that end, we aimed to develop and implement machine learning (ML) and advanced data analysis techniques to standardize and automate the identification of features in TES X-ray spectroscopy data, for calibration, optimization, error identification, and post-processing. We tested several new techniques and found particular success in automating key steps of the calibration of large TES arrays using a method based on derivative dynamic time warping. This method is generic and may be transferable to other types of detector arrays. We investigated ML-based and artificial intelligence (AI)-based techniques, and a framework for interfacing our TES data with common ML software libraries was created.

\begin{tabular}{|l|l|}
\hline LDRD \# 2020-0181 & Innovate \\
\hline Project Title: & $\begin{array}{l}\text { Advanced Method for Ultrahigh Sensitivity and Resolution At-Wavelength X-ray } \\
\text { Optics Characterization }\end{array}$ \\
\hline Investigators: & $\begin{array}{l}\text { Zhi Qiao } \\
\text { Yudong Yao }\end{array}$ \\
\hline
\end{tabular}

\begin{abstract}
The Advanced Photon Source (APS) upgrade is seeking methods to characterize high-quality X-ray optics and wavefront aberration after propagation through a series of components. We offer an alternative choice for high sensitivity optics metrology. Synchrotron facilities use adaptive mirrors to correct small wavefront aberration to achieve smaller focus. This method will be a promising technique to precisely measure the ultra-small wavefront aberration in order to achieve a minimal focal spot. Whereas current wavefront sensing methods use, for example, grating interferometer and speckle tracking based on the geometric displacement, our method is based on far-field diffraction which is more sensitive to wavefront aberration. Our goal was to develop an at-wavelength X-ray optics characterization method, with ultrahigh sensitivity $(<1 / 200$ wavelength) and resolution ( $<0.5$ micrometer), while relaxing requirements on X-ray coherence. We developed a scanning aperture (pinhole) array to achieve far field diffraction and to speed up the scanning process for the wavefront. The small pinhole will also relax the coherence requirement on the X-ray source and reduce the detector-pinhole distance. This method was verified and investigated by numerical simulation and was successfully demonstrated through experiment at the APS.
\end{abstract}




\begin{tabular}{|l|l|}
\hline LDRD \# 2020-0182 & Innovate \\
\hline Project Title: & $\begin{array}{l}\text { In situ Physics-of-Failure Analysis for Wide Bandgap Power Device for } \\
\text { Aerospace and Defense Application }\end{array}$ \\
\hline Investigators: & $\begin{array}{l}\text { Moinuddin Ahmed } \\
\text { Kamel Fezzaa }\end{array}$ \\
\hline
\end{tabular}

\begin{abstract}
Our project mainly focuses on real-time failure analysis of high frequency, high power devices and components. We utilized x-ray high-speed imaging (HSI) at one of Argonne's Advanced Photon Source (APS) beamlines. This work aligns with a general thrust toward an in situ physics-of-failure analysis capability at APS, in this case by testing high-power wide bandgap (WBG) semiconductor devices undergoing accelerated stressing and high-speed switching. More conventional approaches, such as ex situ parametric defect analysis or technology computer aided design (TCAD) simulation with in situ electrical studies, do not adequately deal with defects during operation, especially for the newer generation of WBG device with switching speeds in the gigahertz $(\mathrm{GHz})$ range. The testing protocols we developed accomplish efficient failure analysis and switching fault analysis.
\end{abstract}

\begin{tabular}{|l|l|}
\hline LDRD \# 2020-0194 & Innovate $\quad$ \$24,141 \\
\hline Project Title: & Better Adversarial Machine Learning Through Robust Optimization \\
\hline Investigators: & Matthew Menickelly \\
\hline
\end{tabular}

\begin{abstract}
Given the interest in advancing scientific discovery through (scientific) machine learning, certain elements of popular machine learning systems must be addressed. In particular, this project seeks to make machine learning systems more robust, i.e., less susceptible to adversarial attacks, and more accessible to their users. We developed a novel approach to training machine learning algorithms in the presence of arbitrarily, potentially adversarially, contaminated datasets. Our method is guided by disciplined non-smooth optimization techniques and employs ideas from online optimization to dynamically compute trimmed estimators. We developed and tested both attacking and defending algorithms for use in adversarial training. Various prototypes of our attacking and defending methods were implemented tested on standard machine learning benchmarks throughout the development. Ultimate validation of our approach will arise from applying our adversarial training methods to a scientific machine learning problem and comparing the results to those obtained for the same problem using standardly trained machine learning systems.
\end{abstract}




\begin{tabular}{|l|l|}
\hline LDRD \# 2020-0196 & Innovate $\$ 24,999$ \\
\hline Project Title: & $\begin{array}{l}\text { Artificial Intelligence-based Local Downscaling of Global Coastal Flood } \\
\text { Prediction }\end{array}$ \\
\hline Investigators: & William Pringle \\
\hline
\end{tabular}

\begin{abstract}
The aim of this project was to implement an artificial intelligence (AI)-based downscaling of forecasts of global coastal water levels to city-block-scale coastal flooding predictions worldwide. Whereas this specific goal has not yet been fully realized, our achievements will be critical for continued pursuit of our goal. We supported the dissemination of the results of a study associated with high-resolution projections of coastal water levels in the northeastern region of the United States under a high emissions scenario. In that case, a high-fidelity (50-meter coastal resolution) hydrodynamic storm tide model was forced with three dynamically downscaled regional climate models (RCMs) over three decadal periods (historical, mid-21st century, and late-21st century) to project future changes in peak storm tide elevations at coastal counties in the region. We also modified and tested a water level downscaling computer code (called Kalpana). The code converts coastal water levels to flood depths on high-resolution digital elevation models of topography. It will be critical to obtain simulated predictions of coastal flooding on city-block-scales that can be used for training machine learning (ML) models. Lastly, we contributed to the release of an operational global model, namely the global extratropical surge and tide model of NOAA. Its forecast data can be used for training an ML model that could convert coastal water levels into city-block-scale predictions of flood depths.
\end{abstract}

\begin{tabular}{|l|l|}
\hline LDRD \# 2020-0197 & Innovate $\$ 24,606$ \\
\hline Project Title: & Statistics and Machine Learning to Enhance Reduced Order Models \\
\hline Investigators: & Sudharshan Ashwin Renganathan \\
\hline
\end{tabular}

\footnotetext{
Abstract

High-fidelity models can involve large-scale simulations that take hours or even days for each run. Running many high-fidelity simulations presents a significant computational challenge. Reduced order modeling (ROM) is a technique that can reduce the computational complexity or storage requirement of a computer model, while preserving the expected fidelity with controlled error. Working with lowerorder surrogate models can simplify analysis and control design. Simpler models are also easier to understand and manipulate than high-order models. Our project aimed to improve ROM with the emerging field of machine learning (ML). Existing ROM techniques must be augmented with ideas from statistics and ML, such as optimal experimental design, stochastic process modeling, and deep learning. Specifically, we used deep neural network (DNN) and probabilistic Gaussian process (GP) models to circumvent some of the "intrusive" steps involved in classic ROM techniques, such as projection. Our work demonstrated the ability to speed-up solutions to solve expensive nonlinear partial differential
} 
equations (PDEs) orders of magnitude faster. We demonstrated the utility of our approach in real-world design problems in aerospace engineering.

\begin{tabular}{|l|l|}
\hline LDRD \# 2020-0203 & Innovate \\
\hline Project Title: & $\begin{array}{l}\text { Modeling Dynamics of Complex Chemical Kinetics using Neural Ordinary } \\
\text { Differential Equations }\end{array}$ \\
\hline Investigators: & $\begin{array}{l}\text { Pinaki Pal } \\
\text { Opeoluwa Olawale Owoyele }\end{array}$ \\
\hline
\end{tabular}

\begin{abstract}
Accurate and fast chemical kinetics calculations are key to computational modeling and simulationdriven design of turbulent combustion systems, such as gas turbines and piston engines, for reduced emissions and improved fuel economy. However, the use of high-fidelity detailed chemical kinetic models is prohibitively computation intensive. That has prompted use of reduced-order models that are unfortunately of limited accuracy in practice. This has motivated use of fully connected deep neural networks to predict chemical kinetics as a function of the thermochemical state of the chemically reacting system. Due to the nonlinearities and the multi-scale nature of combustion, these solutions often diverge from the true solutions when deep learning models are coupled with computational fluid dynamics (CFD) solvers. In this project, we developed a novel neural ordinary differential equations (ODE) approach to modeling chemical kinetics. The chemical reaction rates predicted by the neural networks were integrated during training. By backpropagating errors through the ODE solver and the layers of the neural network, the training parameters were adjusted to minimize the difference between predicted and actual ODE solutions. We demonstrated that the proposed approach accurately captures the correct physical behavior and reproduces the results obtained using the full chemical kinetic mechanism at a fraction of the computational cost.
\end{abstract}

\begin{tabular}{|l|l|}
\hline LDRD \# 2020-0204 & Innovate $\quad \$ 17,127$ \\
\hline Project Title: & Silicon and Germanium Delta-Doped Diamond Homoepitaxial Films \\
\hline Investigators: & Nazar Delegan \\
\hline
\end{tabular}

\begin{abstract}
Group-IV complexes, such as silicon-vacancy (SiV) and germanium-vacancy (GeV), hold great promise for quantum communication and networking applications. We planned to expand our diamond film doping capabilities to include group-IV element-vacancy complexes. Our initial goal was creation of GeV quantum defects within optimized, high-quality homoepitaxially grown diamond. Our scheme for incorporating germanium impurities within the diamond matrix allows for the precise control of defect depth, as well as improved control of the crystallographic and isotopic environments. We retrofitted the existing diamond chemical vapor deposition (CVD) tool of Argonne's Materials Science Division to accommodate our proposed process. We thus allowed incorporation into diamond of a Ge precursor
\end{abstract}


(from diluted germanium gas) during diamond growth. Initial Ge-doped high quality diamond samples were synthesized and characterized via secondary ion mass spectroscopy (SIMS) to verify Ge incorporation efficiency as a function of deposition parameters, e.g., as a function of relative mass gas flow rates of the precursors and $\mathrm{CH}_{4}+\mathrm{H}_{2}$ gases. Photoluminescence studies of the GeV quantum centers would be the next step in confirming their successful creation.

\begin{tabular}{|l|l|}
\hline LDRD \# 2020-0208 & Innovate \\
\hline Project Title: & Low-Melting Oxide-Glass: A New Type of Solid-State Electrolyte \\
\hline Investigators: & $\begin{array}{l}\text { Yujia Liang } \\
\text { Joseph A. Libera, Zonghai Chen }\end{array}$ \\
\hline
\end{tabular}

\title{
Abstract
}

We developed a solid-state binder that can enhance the connection between the cathode, lithium-ion carrier, and electron conductor in a Li-ion battery. We have demonstrated this idea by using a mixture of lithium salts (borate-sulfate-carbonate or $\mathrm{Li}_{3} \mathrm{BO}_{3}-\mathrm{Li}_{2} \mathrm{SO}_{4}-\mathrm{Li}_{2} \mathrm{CO}_{3}$ ) as the solid-state binder formed using ultrasonic spray pyrolysis (USP). This material forms our oxide-glass solid state electrolyte (OG-SSE). It showed a melting point under $600^{\circ} \mathrm{C}$. Its low-melting-point and relatively high Li-ion diffusivity are ideal as a solid-state binder for all-solid-state batteries (ASSBs). As a demonstration, we have made composite pellets of solid-state binder and lithium lanthanum zirconium oxide (LLZO, $\mathrm{Li}_{7} \mathrm{La}_{3} \mathrm{Zr}_{2} \mathrm{O}_{12}$ ) and made several comparative measurements of ionic conductivities. They ranged from 0.8 to 3.0 Siemens $/ \mathrm{cm}$. This work solves the issue of lacking an essential component to connect the cathode reactive materials, solid-state electrolyte (Li-ion carrier), and carbon (electron carrier) in ASSBs. We have also addressed the processability of LLZO when it is used as the only component in a battery's separator.

\begin{tabular}{|l|l|}
\hline LDRD \# 2020-0214 & Innovate \\
\hline Project Title: & Advanced Materials for Separations Technology via Electrospinning \\
\hline Investigators: & $\begin{array}{l}\text { Lauren Valentino } \\
\text { Devon Powers }\end{array}$ \\
\hline
\end{tabular}

\begin{abstract}
The production of water and energy rely on separation processes to purify various process streams, making separations integral to water and energy security. Our project focused on the development and characterization of materials fabricated by dual-phase electrospinning for use in electrochemical separation processes. Electrospinning enables a material-by-design approach in which the composition, porosity, and diameter of the fibers can be adjusted depending on the target application. We separately established electrospinning conditions for cation-conductive and anion-conductive nanofibers. These polymer fibers were also co-electrospun to obtain a mixed fiber matrix. The electrospun materials were characterized using ionic conductivity measurements and microscopy techniques. Scanning electron microscopy confirmed the formation of a mixed fiber matrix, and conductivity measurements indicated ion conductivities comparable to commercially available ion exchange (IEX) membranes. We thus
\end{abstract}


demonstrated the feasibility and practical potential of introducing electrospun materials into advanced electrodeionization (EDI) separation technology as well as into other electrochemical separation technologies where IEX resin beads or resin wafers are the current ionic conductors.

\begin{tabular}{|l|l|}
\hline LDRD \# 2020-0218 & Innovate \\
\hline Project Title: & Scalable Bayesian Calibration for Risk-Conscious Building Energy Modeling \\
\hline Investigators: & Qi Li \\
\hline
\end{tabular}

\begin{abstract}
Local, state, and federal government agencies need help in making risk-conscious policy decisions and utilities and building owners need help to improve the cost-effectiveness and reliability of electricity generation, delivery, and consumption. Our plan was to develop realistic case studies to demonstrate a proposed framework. The first would target individual building demand flexibility analysis and would be feasible to run on a desktop computer. Bayesian calibration methods were to be used as a benchmark. The second would apply to community or urban scale building energy management and grid integration analysis. It could demonstrate scalability on a high-performance computer (HPC) platform. Next steps would include application of Gaussian process models and stochastic variational inference algorithms to test and validate approximation performance. That would include investigations on stochastic variational inference algorithms that estimate posterior probability distributions of Bayesian inference through re-parameterization and stochastic optimization. We identified three case study candidates and developed the required working scripts for desktop computer performance benchmarking, the project was terminated at that point when its principal investigator became unavailable.
\end{abstract}

\begin{tabular}{|l|l|}
\hline LDRD \# 2020-0225 & Innovate \\
\hline Project Title: & Evaluation of Precipitation Instrument Performance Across Rain Rates \\
\hline Investigators: & $\begin{array}{l}\text { Adam K. Theisen } \\
\text { Jenni A. Kyrouac, Scott M. Collis, Robert Jackson }\end{array}$ \\
\hline
\end{tabular}

\begin{abstract}
Many instruments are used to measure precipitation ranging from optical to mechanical systems and each has its own benefits and deficiencies. The Atmospheric Radiation Measurement user facility (ARM) Southern Great Plains observatory (SGP) houses over 50 precipitation instruments, using approximately 10 different methods for measurement. The difference in method can lead to a substantial difference in measured rainfall accumulations and rates. Accurate rainfall estimation is crucial to a wide array of applications including radar observations, modeling of climate and hydrological processes and urban impacts. Our study investigated creating a machine learning based adaptive algorithm for obtaining a precipitation best estimate that included situational data (wind speed, temperature, etc.) with SGP data. Our proposed methodology has proved promising, but more work is needed. Therefore, an evaluation best-estimate data product has been provided to the ARM user facility for use and feedback.
\end{abstract}




\begin{tabular}{|l|l|}
\hline LDRD \# 2020-0226 & Innovate \\
\hline Project Title: & $\begin{array}{l}\text { High-Energy X-ray Diffraction and Machine Learning Driven Molecular Dynamics } \\
\text { Simulation Study of Molten Chloride Salts }\end{array}$ \\
\hline Investigators: & $\begin{array}{l}\text { Jicheng Guo } \\
\text { Ganesh Sivaraman }\end{array}$ \\
\hline
\end{tabular}

\begin{abstract}
Molten chloride salts play an important role in concentrated solar power, advanced nuclear reactors and metals production. Understanding the correlation between molten salt structures and their physical properties can provide guidance for designing compositions for their various applications. These salts are composed of mixtures of alkali and alkaline earth chlorides (e.g., $\mathrm{LiCl}, \mathrm{KCl}, \mathrm{NaCl}, \mathrm{MgCl}_{2}$, etc.) and have wide variations in physicochemical properties that are directly correlated with their atomic scale structures. We studied the structure of such salts by combining high energy x-ray diffraction (HEXRD) and machine learning (ML) driven molecular dynamics (MD) simulations. It is anticipated that models can be built to correlate the physical properties and atomic scale structure. When the aspects of structural properties, such as bond length and polarizability that have higher influences on the physical properties are identified, prediction of the key macroscopic observables without the need for challenging experimental measurements of salt properties will be possible. We developed a ML-based interatomic potential for molten $\mathrm{NaCl}$ that can access classical interatomic potential time and length scales while maintaining ab initio precision. Our results outperformed state-of-the-art polarizable models. We also prepared high purity samples of $\mathrm{NaCl}, \mathrm{LiCl}-\mathrm{KCl}$ and $\mathrm{MgCl}_{2}-\mathrm{KCl}-\mathrm{NaCl}$ for $\mathrm{HEXRD}$ experiments at the Advanced Photon Source (APS) to validate simulations. The simulated structure of molten $\mathrm{NaCl}$ did accurately predict physicochemical properties such as diffusion coefficient and conductivity. The methodology developed in this project can be applied to other molten salt systems and can contribute to building a molten salt structure database for many applications.
\end{abstract}

\begin{tabular}{|l|l|}
\hline LDRD \# 2020-0229 & Innovate \\
\hline Project Title: & $\begin{array}{l}\text { Developing Cathodes with Advanced Surface Treatment Towards Future } \\
\text { Electron Sources }\end{array}$ \\
\hline Investigators: & Jiahang Shao \\
\hline
\end{tabular}

\begin{abstract}
Electron sources are crucial components for experimental studies in the atomic and molecular sciences. Advanced radio frequency (RF) photocathode gun sources will require higher beam brightness (i.e., high gradient operation). We aimed to demonstrate a novel surface treatment method to suppress field emission current in high gradient radio-frequency structures. That method is high-power impulse magnetron sputtering (HiPIMS). We demonstrated that HiPIMS is an effective way to achieve high surface gradient through complete removal of surface defects. Success criteria are lower RF breakdown
\end{abstract}


rate and lower dark current level with unchanged thermal emittance and quantum efficiency compared to untreated surfaces. Three cathodes were tested in the L-band ( $1565 \mathrm{~nm}$ to $1625 \mathrm{~nm}$ ) photocathode gun test-stand at the Argonne Wakefield Accelerator (AWA). They included an untreated cathode exposed to high field, a treated cathode exposed to high field, and an untreated cathode at low field as a control to account for background emission from the gun itself. It was found that background emission contributed a large portion of the total observed field emission. After background subtraction, the treated cathode showed up to a $75 \%$ reduction in field emission at surface fields up to $\sim 120 \mathrm{MV} / \mathrm{m}$. Future experiments should be conducted with an in situ field emission imaging system to systematically study the effectiveness of our surface treatment method.

\begin{tabular}{|l|l|}
\hline LDRD \# 2020-0233 & Innovate \\
\hline Project Title: & $\begin{array}{l}\text { Advanced Algorithms for Optimizing Critical Infrastructure Systems Affected by } \\
\text { High-Impact Rare Events }\end{array}$ \\
\hline Investigators: & $\begin{array}{l}\text { Anirudh Subramanyam } \\
\text { Vishwas Rao }\end{array}$ \\
\hline
\end{tabular}

\begin{abstract}
Critical infrastructure systems such as electric power, water, and natural gas networks are vulnerable to high-impact rare events such as natural disasters, cyber attacks, and cascading failures leading to catastrophic blackouts. Costs of such events can exceed $\sim$ billion. The rarity and criticality of these events, coupled with the paucity of real-world data, render existing algorithms for the optimization and simulation of such systems inadequate. Decision makers and operators need a tool that more conveniently addresses risk quantification, computing the probability (and associated risk) of an extreme high-impact event accurately and efficiently for an arbitrary design and operational configuration of an infrastructure network. The tool must also incorporate a risk mitigation algorithm that incorporates the risk computations in a computationally efficient and scalable decision-making framework to determine optimal design and operational configuration of the network. We have developed those new methodologies, by combining mathematical optimization techniques with modern statistical tools, enabling accurate quantification and subsequent mitigation of high-impact rare events in electric power systems and structural engineering. Our project has provided non-traditional insights into designing robust networks that systematically trade off system reliability against cost efficiency, and contributed fundamental advances to the relatively unexplored areas of optimization and uncertainty quantification of rare events.
\end{abstract}




\begin{tabular}{|l|l|}
\hline LDRD \# 2020-0173 & Swift \\
\hline Project Title: & Interlayer Films for Solid State Batteries \\
\hline Investigators: & Jeffrey W. Elam \\
\hline
\end{tabular}

\begin{abstract}
Solid-state batteries (SSBs) offer higher capacity and improved reliability and safety compared to conventional lithium-ion batteries and may displace lithium-ion batteries in portable electronics, electric vehicles, and other applications. However, undesirable chemical and physical processes at the interfaces between the electrodes and the electrolyte have been a development challenge. Interlayer films deposited at these interfaces could solve these problems. We developed atomic layer deposition (ALD) methods to deposit thin films of lithium ion conducting materials, specifically lithium phosphate and lithium phosphorus oxynitride, to be used as interlayers in SSBs. Films were created and characterized from an array of precursor chemistries and atomic layer deposition parameters including temperature. Spectroscopic ellipsometry and X-ray photoelectron spectroscopy measurements on the films were facilitated through use of silicon $(\mathrm{Si})$ substrates. Thickness, refractive index and composition were thus obtained. We utilized interdigitated comb substrates to facilitate ionic and electronic conductivity measurements. In summary, we discovered an ALD process that enables low temperature growth of the high purity and ultrathin ionically conducting coatings.
\end{abstract}

\begin{tabular}{|l|l|}
\hline LDRD \# 2020-0250 & Swift \\
\hline Project Title: & $\begin{array}{l}\text { High-Fidelity Multiphase Simulation of Steam Separators in a Light Water } \\
\text { Reactor }\end{array}$ \\
\hline Investigators: & $\begin{array}{l}\text { Lander Ibarra } \\
\text { Dillon R. Shaver }\end{array}$ \\
\hline
\end{tabular}

\begin{abstract}
This project demonstrates how computational fluid dynamics modeling will aid the design of a steam separator experimental facility. We assessed and quantified the limitations of the high fidelity two fluid model in Nek-2P, the extension to two-phases Argonne's NEK5000 computational fluid dynamics (CFD) code. We calculated the distribution of steam and droplet volume fractions in a representative boiling water reactor (BWR) cyclone-type steam separator. Several areas of improvement were identified, notably in the numerical behavior of the algorithms. We documented the assessment of the Nek-2P closure models to simulate the phasic separation for a representative steam separator geometry and outlined the implementation of additional models necessary to calculate the separated liquid fraction returning to the reactor coolant cycle. Ideally, multiple flow conditions can now be modeled with Nek$2 \mathrm{P}$, e.g., off-rated operation and increased core flow (ICF) conditions, to determine the effects on buoyancy and inertia and characterize these effects on vapor quality and droplet flow paths.
\end{abstract}




\begin{tabular}{|l|l|}
\hline LDRD \# 2020-0252 & Swift \\
\hline Project Title: & Slurry Based Flame Spray Pyrolysis for Catalyst Synthesis and Production \\
\hline Investigators: & $\begin{array}{l}\text { Rongyue Wang } \\
\text { Theodore R. Krause, Joseph A. Libera }\end{array}$ \\
\hline
\end{tabular}

\begin{abstract}
Flame Spray Pyrolysis (FSP) is a commercial process to produce metal oxide materials. Metal oxide supported precious metal catalysts can be prepared with this process. However, a large portion of precious metal is buried inside metal oxide and is therefore wasted. We pursued a single-step FSP synthesis process for metal-supported catalysts that provides better control for depositing the metal nanoparticles on the oxide surface by co-feeding the precursors for producing the metal nanoparticles with pre-formed metal oxide particles as a slurry. Slurry-based FSP for catalyst synthesis can improve catalyst performance and it is also applicable to other areas where coated nanoparticles are needed. Development of slurry formulations requires determination of the most economic combination of combustible solvents as well as organic surfactants to achieve stable slurries with controllable combustion properties. Study is required of the effect of slurries' compositions on slurry particle properties after processing to optimize the support phase properties. A catalyst synthesized with this new slurry FSP process has most of the precious metal on the surface of a metal oxide support and that makes FSP a viable process for metal oxide supported precious metal catalyst production. In future projects, a suitable catalytic reaction should be used as a demonstration of the improved catalytic properties and additional characterization of catalyst samples performed.
\end{abstract}

\begin{tabular}{|c|c|}
\hline LDRD \# 2020-0253 & $\$ 50,063$ \\
\hline Project Title: & $\begin{array}{l}\text { Adaptive-Scale Multivariate Semi-Analytical Computation Approach for } \\
\text { Transient Simulation of Networked Energy Systems in National Resilience } \\
\text { Analysis }\end{array}$ \\
\hline Investigators: & $\begin{array}{l}\text { Rui Yao } \\
\text { Feng Qiu }\end{array}$ \\
\hline
\end{tabular}

\begin{abstract}
Networked energy systems (NES), such as electric power grids and natural gas networks, comprise the backbone of our energy infrastructure. Accurate, efficient, and robust transient simulation of NES at a national level is needed. With this project, we intended to advance the computational methodology for NES analysis. A multivariate semi-analytical solution (MV-SAS) approach has shown promising performance for high-order and exceptionally adaptive-scale simulations in solving various NES analysis tasks. NES transient analysis relies on solving large-scale partial differential equations (PDEs), a challenging task even for the current state-of-the-art commercial solvers. We have developed the MVSAS approach for solving PDEs and applied it to natural gas pipeline transient analysis. Compared with conventional approaches, ours has demonstrated advantages in both solution accuracy and
\end{abstract}


computational speed. Our novel approach can become the next-generation computational approach for solving PDEs of multi-scale, complex transient processes of large-scale NES. This tool will also help improve the resilience of energy systems.

\begin{tabular}{|l|l|}
\hline LDRD \# 2020-0269 & Swift \\
\hline Project Title: & Adsorption Strategies for PFAS \\
\hline Investigators: & $\begin{array}{l}\text { Edward F. Barry } \\
\text { John Quinn }\end{array}$ \\
\hline
\end{tabular}

\title{
Abstract
}

We investigate removal strategies for per- and polyfluoroalkyl substances (PFAS) from aqueous solutions using adsorbent platforms based on the OleoSponge and EZSelect technologies. We seek to validate Argonne's flexible nanostructured materials as selective and reusable adsorbents for the capture of PFAS in water. PFAS are of growing concern for their potentially toxic effects and extreme persistence in the environment and the human body. Even minute concentrations can have damaging effects. Current approaches for treating PFAS-contaminated water rely on granular activated carbon (GAC) or ion exchange (IX) resin. While these technologies can be effective on small volumes, they are lowthroughput, produce solid waste (especially for GAC), have clogging issues, carry high maintenance costs (especially for IX), and both are not able to remove key targeted contaminants from the water. Our intent is to demonstrate proof-of-concept for an innovative and cost-effective treatment of PFAS in water. Building on the success of reusable sorbent materials such as OleoSponge, our efforts focused on tuning material surface chemistry, as well as engineering sorption mechanisms based on hydrophobic effects, charge-based mechanisms, and combinations thereof, to target PFAS related compounds. We developed and tested a number of synthesis methods for adsorbents and adsorption strategies. Materials were synthesized and characterized using advanced characterization tools. Three different candidates were tested and subsequently optimized based upon their surface chemistry, material porosity, and surface area.

\begin{tabular}{|l|l|}
\hline LDRD \# 2020-0270 & Swift \\
\hline Project Title: & $\begin{array}{l}\text { Molecular Engineering of Field-effect Transistor Sensor for Ultrasensitive } \\
\text { Detection of Per- and Polyfluoroalkyl Substances (PFAS) in Water }\end{array}$ \\
\hline Investigators: & $\begin{array}{l}\text { Junhong Chen } \\
\text { Stuart Rowan }\end{array}$ \\
\hline
\end{tabular}

\begin{abstract}
We sought to develop a field-effect transistor (FET) sensor featuring fast response, high sensitivity and selectivity, and ease of operation for in situ detection of per- and polyfluoroalkyl substances (PFAS) in water. PFAS contamination is an ecological and human health concern. Our goal was demonstration of detection of PFAS in water at the level of 70 nanograms/liter (ng/L). Our approach was to molecularly engineer cyclodextrin- (CD) containing polymeric materials for specific binding with PFAS. The FET
\end{abstract}


sensor would create opportunities for inline monitoring of water quality and offer an effective strategy for risk management through identification and quantification of PFAS in water. Three strategies were proposed for linking beta-cyclodextrins $(\beta-C D)$ to gold nanoparticles decorating the FET. Two strategies were explored for thio-beta-cyclodextrins $(\mathrm{SH}-\beta-\mathrm{CD})$ synthesis in gram scale. Nuclear magnetic resonance spectroscopy (NMR) was used to identify its formation after purification and recrystallization. A background literature review was completed regarding PFAS adsorbents (especially cyclodextrin-based adsorbents), PFAS sensors, and characterization tools used to study PFAS-probe interactions. We found both electrostatic attraction and fluorine-fluorine interaction explored for PFAS sensing and removal. For characterizing our PFAS- $\beta-C D$ interactions, isothermal titration calorimetry (ITC), NMR, surface plasmon resonance (SPR), and molecular docking together with Argonne's Advanced Photon Source (APS) were appropriate tools.

\begin{tabular}{|l|l|}
\hline LDRD \# 2020-0271 & Swift \\
\hline Project Title: & Interoperability and Cybersecurity Assessment of Smart Charge Adapter \\
\hline Investigators: & $\begin{array}{l}\text { Daniel S. Dobrzynski } \\
\text { Roland Varriale, Nick Lau }\end{array}$ \\
\hline
\end{tabular}

\footnotetext{
Abstract

Plug-in electric vehicles (PEVs) are rising in popularity. They can create synergies between electrified transport and utility power systems. These synergies include reduction of load impact on the grid and the increased utility of renewable energy via Smart Charge Management (SCM) systems. Interoperable and cyber-secure components are key for a SCM system to operate reliably. We focused on validating the interoperability and cybersecurity of an existing Argonne Smart Charge Management technology, the Smart Charge Adapter (SCA), for wide scale application. The SCA is intended to provide direct benefits to electricity grid operators, energy service providers, charging network providers, and PEV owners. The SCA is exchanging critical information from the PEV to the charging infrastructure; thus evaluating the SCAs communication protocol implementation is critical. The direct ties between the electricity grid, the SCA, and transportation systems make the technology a potential vulnerability to national security. We therefore included vetting the SCA from a cybersecurity perspective in our project. Although the protocol itself is sound, the implementation details may introduce gaps in security that could manifest as vulnerabilities which can compromise data confidentiality, integrity, and availability. We purchased a Keysight Charge Discovery System (CDS) for testing the SCA in development, and we were trained in its use. It has been helpful in verifying functionality for SCA devices, twelve of which were paired with PEV owners as a pilot program.
} 


\begin{tabular}{|l|l|}
\hline LDRD \# 2020-0279 & Swift \\
\hline Project Title: & $\begin{array}{l}\text { Manufacture of Eco-Friendly Biomass-Based Plastic with Highly Reusable, } \\
\text { Recyclable and Reproducible Properties }\end{array}$ \\
\hline Investigators: & $\begin{array}{l}\text { Chia-Wei Hsu } \\
\text { Krzysztof Z. Pupek }\end{array}$ \\
\hline
\end{tabular}

\title{
Abstract
}

We utilize cellulose and polyurea to produce a new type of biomass-based polymer featuring high recyclability and reusability with high reproduction quality. Cellulose is the abundant polymer from plants and it has high thermal stability for further applications. Cellulose nanocrystals (CNC) feature similar properties to carbon fiber and can produce superior construction material with other composite polymers. Previously, an efficient method to extract CNC from plants was developed. We have modified the CNC structure by inserting isocyanate from polyurea. Free isocyanate from the reversible steric urea bond in the polyurea can react with cellulose to form branched polymeric networks. Based on the temperature of reaction and the amount of polyurea added, we can adjust the degree of crosslinking in the cellulose by manipulating the equilibration between the urea and carbamate bonds. Furthermore, we can reproduce this plastic with lower melting point based on the reversible cross-linking formation and even modify the chain strength with different additives. Consequently, this cellulose-based polymer can lead to cost-effective recycling processes and energy-preserving reusability. We revealed the optimal condition of the synthesis of cellulose-carbamate polymer that features different properties from the cellulose. There were three kinds of novel cellulose-based polymers obtained. Also, polyethylene terephthalate (PET) may be depolymerized and inserted into cellulose to create a novel PET-cellulose polymer. This leads to an upcycling of post-consumer PET to generate a biomass-based plastic. Consequently, this cellulose-based polymer can lead to cost-effective recycling processes and energy-preserving reusability. Such results pave the way toward the development of highly recyclable plastics from either biomass feedstock or post-consumer plastics.

\begin{tabular}{|l|l|}
\hline LDRD \# 2020-0281 & Swift \\
\hline Project Title: & Surrogate Fallout for Rad-Nuke Resuspension and Decontamination Studies \\
\hline Investigators: & $\begin{array}{l}\text { Michael D. Kaminski } \\
\text { Derek R.McLain }\end{array}$ \\
\hline
\end{tabular}

\begin{abstract}
We are developing several methods of synthesizing morphologically (i.e., chemically-, mineralogicallyand physically) accurate surrogate nuclear and radioactive fallout particles. The surrogates permit accurate fate and transport experiments and specifically allow for the development of evidence-based decontamination methods for an expedited environmental recovery. We produced metal and concrete based fallout using high temperature melting techniques with a bench-scale electric arc furnace to form irregularly shaped composite materials. We also used wet chemistry precipitation techniques to form uniform microspheres with radioactive and fluorescent tags to facilitate monitoring. We characterized the off-gas particulate and melt for a variety of starting materials. Tests began with pure metals (iron,
\end{abstract}


zirconium) and then alloys (steel, zircaloy) before testing ceramics (cement, concrete). These were meant to mimic nuclear reactor and urban sources. The melt materials were studied by various microscopy techniques to determine their microstructures and compositions. Also, we were able to identify the gaps in modeling the resuspension of fallout.

\begin{tabular}{|l|l|}
\hline LDRD \# 2020-0284 & Swift \\
\hline Project Title: & Two-Dimensional Numerical Modeling of Tailings Dam Failures \\
\hline Investigators: & $\begin{array}{l}\text { Mustafa Siddik Altinakar } \\
\text { Kyle B. Pfeiffer }\end{array}$ \\
\hline
\end{tabular}

\begin{abstract}
Argonne collaborates with the Federal Emergency Management Agency (FEMA) on preparedness, emergency response planning, disaster resilience, dam safety and regional risk assessment areas. The DSS-WISE ${ }^{\mathrm{TM}}$ (Decision Support System for Water Infrastructural Security) code provides automated dam-break flood modeling capability to FEMA and other users. We have developed a new module, DSS-WISE ${ }^{\mathrm{TM}}$ Tail, that extends the applicability of the DSS-WISE ${ }^{\mathrm{TM}}$ to simulate and estimate consequences of tailings dam failures, which have a much higher risk of failure than that of conventional water storage dams. Moreover, our module can later be extended to develop tools to simulate other types of geophysical hazards such as landslides, debris flows, mudflows, and lahars. Ours is a two-dimensional numerical module for simulation of non-Newtonian slurry flows by solving a non-conservative form of two-dimensional shallow water equations. The numerical engine solves full dynamic shallow water equations using finite volume discretization over a regular Cartesian grid derived directly from digital elevation data. Because tailings flows are non-Newtonian flows of sediment-water mixtures, shallow water equations were computed using a rheological model. In addition to roughness of the land cover, that model accounted for cohesive yield stress, apparent viscosity, and turbulent and dispersive shear stress. The numerical model was verified using laboratory test data and validated using field data from past tailings dam failures.
\end{abstract}

\begin{tabular}{|l|l|}
\hline LDRD \# 2020-0286 & Swift \\
\hline Project Title: & $\begin{array}{l}\text { Incorporating Artificial Intelligence Capabilities to Support Critical Infrastructure } \\
\text { Interdependency Analysis }\end{array}$ \\
\hline Investigators: & $\begin{array}{l}\text { Lawrence Lewis } \\
\text { Prasanna Balaprakash }\end{array}$ \\
\hline
\end{tabular}

\begin{abstract}
Promoting a secure and resilient electric grid and energy sector is only possible by understanding and modeling complex systems interdependencies. A better understanding of the resilience requirements of all critical infrastructure sectors and definition of how the energy sector plays a central role for the security and resilience of these sectors is needed. Helping to satisfy that need would be an infrastructure dependency database developed using artificial intelligence (AI) and machine learning
\end{abstract}


$(\mathrm{ML})$. Our intent was to develop that Al-compatible database and use it to train an artificial intelligence application that makes predictions across network analysis products. However, it became clear that a foundational step toward incorporating Al into our models would be to first develop an optimization model, and that is primary product of this project. This model reorganizes inputs and outputs into a non-GIS (geographic information system) format that would enable future Al incorporation. The model is built on an infrastructure hypergraph structured to enable real-world data to be ingested efficiently beyond a purely GIS approach and toward an Al-compatible database schema. It provides quantitative probabilistic modeling of service connection networks with expanded attributes as well as simulation of propagation of cascading failures across interdependent infrastructure. Our optimization model represents a significant advancement of our analytic capabilities and is a first step toward future Al incorporation.

\begin{tabular}{|l|l|}
\hline LDRD \# 2020-0290 & Swift \\
\hline Project Title: & Identify and Catalog Information Sources for Global Energy Security Database \\
\hline Investigators: & $\begin{array}{l}\text { Duane R. Verner } \\
\text { Bruce Hamilton }\end{array}$ \\
\hline
\end{tabular}

\section{Abstract}

The energy security of international allies and partners is a key United States foreign policy objective. Energy diversification and interconnectivity among nations are crucial factors in this regard. A comprehensive database that catalogs relevant information sources will allow modeling and assessment of global energy systems. We established an energy security data catalog that enables users, from modelers to analysts and data scientists, to efficiently discover and consume data sources from countries and regions of focus. We began with a pilot project for Central and Eastern Europe. As part of this project, we launched the Global Energy Security Testbed (GEST) at Argonne that enables access to leading edge expertise, research facilities, and a virtual testbed for modeling complex infrastructure systems.

\begin{tabular}{|l|l|}
\hline LDRD \# 2020-0291 & Swift \\
\hline Project Title: & $\begin{array}{l}\text { Millifluidic Optical Cell to Measure Uranium Hexafluoride Hydrolysis Reaction } \\
\text { Dynamics }\end{array}$ \\
\hline Investigators: & $\begin{array}{l}\text { Cari A. Launiere } \\
\text { Nicholas J. Condon, Peter A. Kozak }\end{array}$ \\
\hline
\end{tabular}

\section{Abstract}

A thorough understanding of uranium hexafluoride $\left(\mathrm{UF}_{6}\right)$ hydrolysis is needed to enable new technologies that rely on the detection or characterization of uranium hexafluoride hydrolysis products for safeguards verification, proliferation detection, and nuclear safety. Whereas end products of the complete hydrolysis reaction are hydrogen fluoride (HF) and a uranium-fluoride compound called uranyl fluoride $\left(\mathrm{UO}_{2} \mathrm{~F}_{2}\right)$, several intermediate reaction products occur. Past work on the kinetics of uranium 
hexafluoride hydrolysis is not adequate in that it relies on experiments limited by outdated techniques and relatively poor experimental design. Design of a new analytical tool for gas phase reaction monitoring is needed. We developed a finite volume model to optimize experimental design for Raman spectroscopy reaction kinetics measurements in a millifluidic device. It describes the set of conditions under which a moist air stream with variable moisture levels and a uranium hexafluoride plus argon gas stream will be well-mixed in a millifluidic channel on a time scale of less than one second. The model characterizes the degree of mixing of the two gases as a function of time and distance, as well as the rate of back-diffusion of air towards the uranium hexafluoride source. Our model was used to identify the geometries and flow conditions under which reaction kinetics measurements can be decoupled from gas mixing rates.

\begin{tabular}{|l|l|}
\hline LDRD \# 2020-0298 & Swift \\
\hline Project Title: & Enhancing Capabilities of X-ray Diagnostics for Aerospace Propulsion \\
\hline Investigators: & $\begin{array}{l}\text { Brandon A. Sforzo } \\
\text { Christopher Powell }\end{array}$ \\
\hline
\end{tabular}

\begin{abstract}
We were interested in preparation for experiments focusing on measurement of two-phase flows using $x$-ray diagnostics to examine aero-spray injectors and sprays at aeroengine relevant conditions, necessitating the infrastructure to reach elevated temperatures and pressures, with a compatible chamber and geometries of interest. We have conceptualized a multifaceted program as follows. Hard $x$-ray tomography would characterize as-built nozzle hardware for understanding the influence of geometric variability. Highspeed x-ray imaging would resolve internal fluid dynamics and near-nozzle behavior, exhibiting the dynamic behavior of fluid breakup and qualitative structure. Time-resolved $x$-ray radiography would provide quantitative measurements of fluid mass distribution and spatial asymmetry though tomographic techniques. Our experimental observations and validation of simulation results would deliver new data, physical insight, models and experimental techniques that improve: (1) Emissions predictions through improved fuel distribution models, (2) design flexibility for physical property variability in alternative fuels, and (3) dynamic models between thermo-acoustic behavior and liquid fuel delivery. Furthermore, the facility for detailed $x$-ray diagnostics and linkage to modeling workflows would be available for measurements on manufacturer-specific hardware and conditions. This concept development project, along with our expertise in data collection, analysis and workflows, demonstrated our in-house capabilities. Design and set up of some early experimental facilities were also initiated.
\end{abstract}




\begin{tabular}{|l|l|}
\hline LDRD \# 2020-0300 & Swift \\
\hline Project Title: & $\begin{array}{l}\text { Dichroic Ptychography for Mapping of Magnetic Domains and Orbital } \\
\text { Anisotropies }\end{array}$ \\
\hline Investigators: & $\begin{array}{l}\text { Joerg Strempfer } \\
\text { Junjing Deng }\end{array}$ \\
\hline
\end{tabular}

\title{
Abstract
}

New approaches to imaging domains and domain walls of magneto-electric materials, showing metal-insulator transitions with varying temperature, will provide a new and deeper understanding of quantum materials and will help improve design of nano-structured tailored materials with specific properties. Dichroic ptychography coherent diffraction imaging (CDI) is such a new approach. The implementation of this capability at the Advanced Photon Source (APS) will offer new scientific possibilities, especially after the APS upgrade when coherent beam flux will be two orders of magnitude greater than today. For reconstruction of real-space magnetic densities and electronic orbital anisotropies in three dimensions, a nano-positioning stage for precise sample positioning has been incorporated at APS beamline 4-ID-D together with zone plate focusing optics. We proved the successful implementation of the setup with the first structural and magnetic reconstruction using polarization-dependent CDI with circular polarization control on a thin film magnetic sample. Results from this ferromagnetic test structure with 2D dichroic transmission ptychography will be used to refine and develop the software for image reconstruction. This enabled the next efforts for the implementation of a reference standard for the coherent dichroic flux for the future Polarization Modulation Spectroscopy (Polar) beamline after the APS upgrade and the development of appropriate and specialized reconstruction algorithms.

\begin{tabular}{|l|l|}
\hline LDRD \# 2020-0303 & Swift \\
\hline Project Title: & $\begin{array}{l}\text { Artificial Intelligence Driven Development of a Compact Antimicrobial } \\
\text { Resistance Prediction Platform Using the Cloud Lab }\end{array}$ \\
\hline Investigators: & James J. Davis \\
\hline
\end{tabular}

\begin{abstract}
Antimicrobial resistance (AMR) is a growing impediment to the treatment of bacterial infections. Antimicrobial susceptibility testing determining which antibiotics will be effective against bacterial pathogens requires isolation and culturing which takes over 24 hours for even the fastest growing pathogens. Immediate point of care polymerase chain reaction (PCR) testing has failed to gain full adoption because the genes conferring resistance are too numerous. Artificial intelligence (Al) approaches can tackle this problem by identifying the smallest number of genomic regions necessary for differentiating susceptible and resistant strains with the highest possible accuracy and therefore make more tractable the selection of a set of PCR primers for predicting AMR phenotypes. We used an experimental design where the PCR primers are systematically generated and evaluated in the cloud lab using a process controlled by an Al-based evaluation of the viable primer set. Potential PCR primers from
\end{abstract}


approximately 1700 clinical Klebsiella pneumoniae strains were developed in silico. Using these primers as input, a machine learning (ML) model was developed to predict susceptibility or resistance to approximately 20 antibiotics. The set of potential primers were then synthesized at Integrated DNA Technologies, Inc. (IDT) and shipped to Emerald Cloud Lab (ECL) for automated evaluation over the genomic DNA from a representative collection of about 200 Klebsiella clinical isolates. After determining the appropriate reaction conditions, we were able to perform PCR reactions for the top seven primer pairs in triplicate over the $\mathbf{2 0 0}$ genomic DNA samples. We are comparing the accuracy of the differential amplification versus the in-silico model results.

\begin{tabular}{|l|l|}
\hline LDRD \# 2020-0304 & Swift \\
\hline Project Title: & $\begin{array}{l}\text { Develop an Agent-Based Model Applicable for Simulating COVID-19 Disease } \\
\text { Propagation and Interventions in an Urban Area }\end{array}$ \\
\hline Investigators: & $\begin{array}{l}\text { Charles M. Macal } \\
\text { Jonathan Ozik }\end{array}$ \\
\hline
\end{tabular}

\section{Abstract}

This project extends our knowledge in agent-based modeling decision science to address the current national impact of COVID-19. Our CityCOVID model is specifically and uniquely geared for fine-grained spatiotemporal infectious disease modeling of urban and regional environments and uses the City of Chicago as a test bed. We are generalizing the simulation and associated datasets to be applicable to any U.S. city or urban area. We built the agent-based epidemiological model and the workflows to allow it to run on high-performance computing (HPC) platforms. CityCOVID specifically forecasts COVID-19 spread in Chicago. It mimics the movement and activities, contacts, and disease transmission pathways for Chicago's 2.7 million residents. It models individual agents and the protective measures they might adopt (social distancing, mask wearing, choosing vaccination), how they might react to acquiring COVID19 , as well as how they might react to public health messaging. We ran the model to forecast COVID-19 spread for a variety of scenarios and tested possible interventions before they are implemented by public health officials, providing forecasts to the Chicago Department of Public Health (CDPH) of new cases, hospitalizations, ICU needs, and the COVID-19 reproductive number since March 2020. Those data were also provided to the Illinois Governor's Modeling Task Force for COVID Region 11, Chicago. In collaboration with CDPH, we identified the key questions being asked by public health officials as they arise and developed an evidence-based scientific process that uses CityCOVID to generate timely answers for decision makers. CityCOVID is unique among the models being applied to Chicago and other cities in several way: its highly granular level of detail used to represent the agents (people surrogates), their locations, their hourly activities, their contacts with other agents, and possible behaviors in response to the disease and public health messaging. Our model was recognized as a finalist for the Association for Computing Machinery's Gordon Bell Special Prize for High Performance ComputingBased COVID-19 Research. 


\section{Argonne}

Laboratory Directed Research and Development

Argonne National Laboratory

9700 South Cass Avenue, Bldg. 201

Argonne, IL 60439

www.anl.gov 